FZJ-IKP(TH)-1999-03

UWThPh-1999-15

\title{
Virtual photons in baryon chiral perturbation theory
}

\author{
Guido Müller地, Ulf-G. Meißner地 \\ *Universität Wien, Institut für Theoretische Physik \\ Boltzmanngasse 5, A-1090 Wien, Austria \\ ${ }^{\ddagger}$ Forschungszentrum Jülich, Institut für Kernphysik (Theorie) \\ D-52425 Jülich, Germany
}

\begin{abstract}
We construct the general Lagrangian for relativistic and heavy baryon chiral perturbation theory with virtual photons to fourth order. We work out the electromagnetic and strong isospin violating contributions to the nucleon self-energy, the nucleon mass and the scalar form factor of the nucleon. Electromagnetic effects for the shift to the Cheng-Dashen point can be as large as $2 \mathrm{MeV}$. We also discuss the corrections to Weinberg's prediction for the scattering length difference $a\left(\pi^{0} p\right)-a\left(\pi^{0} n\right)$ and show that they are small.
\end{abstract}

${ }^{\# 1}$ Work supported in part by TMR, EC-Contract No. ERBFMRX-CT980169 (EURODA $\Phi$ NE).

\#2 email: gmueller@doppler.thp.univie.ac.at

\#3 email: Ulf-G.Meissner@fz-juelich.de 


\section{Introduction}

There has been renewed interest in precisely calculating electromagnetic (em) corrections to low energy strong and semi-leptonic processes involving the (pseudo) Goldstone bosons of the strong interactions. For example, the two loop calculations for elastic pion-pion scattering in the framework of chiral perturbation theory, which is the effective field theory (EFT) of the Standard Model at low energies, have now reached such a precision that it is mandatory to also evaluate the pertinent dual effects of virtual photons. Furthermore, to systematically investigate the violation of isospin symmetry one has to account for its two competing sources consistently, namely the light quark mass difference $m_{u}-m_{d}$ as well as the virtual photon effects. Efforts to include virtual photons in the mesonic effective chiral Lagrangian to and beyond leading order based on an extended power counting and to calculate consequences for a variety of processes can be found e.g. in refs. [1]-[6].

Baryon chiral perturbation theory offers another possibility of investigating isospin violation. As first stressed by Weinberg [7], reactions involving nucleons and neutral pions can lead to gross violations of isospin, e.g. in the scattering length difference $a\left(\pi^{0} p\right)-a\left(\pi^{0} n\right)$ he predicted an effect of the order of $30 \%$. This is because chiral symmetry and isospin breaking appear at the same order and the leading isospin symmetric terms involving neutral pions are suppressed due to chiral symmetry. This calculation was sharpened and extended also to the so-called pion-nucleon $\sigma-$ term in ref. [8] (which we call "MS" from here on). In that paper the complete effective chiral pion-nucleon Lagrangian in the presence of virtual photons to one loop and third order in small momenta was constructed. These considerations were extended to other threshold pion-nucleon channels in [9]. It is, however, known that precise and complete one loop calculations in the baryon sector should be carried out to fourth order for two reasons. First, the chiral expansion in small momenta and meson masses proceeds in steps of one power in the presence of baryons (unlike in the meson sector, were only even powers in small momenta or meson masses are allowed for a large quark condensate), so that a complete one loop calculation should include terms of orders $q^{3}$ and $q^{4}$ (where $q$ collectively denotes the small expansion parameters). Second, it has also been shown that in many cases one loop graphs with exactly one dimension two insertion are fairly large (which is related to the fact that the corresponding coupling constants encode the leading effects due to the close-by $\Delta(1232)$ resonance [10]). These terms are of $\mathcal{O}\left(q^{4}\right)$. It has also been argued that the chiral expansions in odd and even powers should be considered separately, i.e. a one loop calculation to third order only gives the leading corrections to the dimension one tree graphs.

Most calculations in baryon CHPT are performed in the so-called heavy fermion formulation [12, 13], which is called heavy baryon chiral perturbation theory (HBCHPT). This is based on the observation that a straightforward extension of CHPT with baryons treated fully relativistically leads to a considerable complication in the power counting since only nucleon three-momenta are small compared to typical hadronic scales, as discussed in detail in ref. [14]. Another advantage of the heavy fermion formulation is its extreme computational simplicity, since all Dirac $\gamma$-matrices are expressed in terms of the nucleons four-velocity and spin vector. However, one has to be careful with strict non-relativistic expansions since in some cases they can conflict strictures from analyticity, as discussed e.g. in ref. [15]. Therefore, in ref.[16], a novel scheme was proposed 
which is based on the relativistic formulation but circumvents the power counting problems (to one loop) by a clever separation of the loop integrals into IR singular and regular parts. In this formulation, all analytic constraints are fulfilled by construction. We remark, however, that the calculational simplicity of the heavy fermion approach is lost. For comparison, neutral pion photoproduction off nucleons calculated relativistically involves some 63 loop graphs [17] to $\mathcal{O}\left(q^{3}\right)$ but only 4 to the same order in HBCHPT [18]. For these reasons, we give the effective Lagrangian with virtual photons in the relativistic as well as in the heavy baryon formulation. In any case, in a systematic construction of the generating functional for HBCHPT, one should start from the fully relativistic theory [13].

The manuscript is organized as follows. In section 2, we construct the fourth order pionnucleon Lagrangian with virtual photons. First, we write down the pertinent building blocks and construction principles. Then, we summarize what is known about the first three orders of the effective Lagrangian and then give explicitely the fourth order terms, both in the relativistic and the heavy baryon formulation. Some applications are discussed in section 3. First, we consider the nucleon self-energy and mass shift including also photon loops (as demanded by consistency). To deal with the IR divergences stemming from the masslessness of the photon, we introduce a small photon mass $m_{\gamma}$ and additional "photonic" counterterms $\sim m_{\gamma}^{2}$. Their effect cancels in physical observables but these terms need to be retained in intermediate steps. We then consider the isospin-violating corrections to the nucleon scalar form factor $\sigma_{\pi N}(t)$, more precisely we are concerned with the shift to the Cheng-Dashen point, $\sigma_{\pi N}\left(2 M_{\pi^{0}}^{2}\right)-\sigma_{\pi N}(0)$. As a third application, we work out the corrections to Weinberg's famous prediction concerning the S-wave scattering lengths difference for neutral pions off nucleons, $a\left(\pi^{0} p\right)-a\left(\pi^{0} n\right)$, which to third order is given entirely in terms of the light quark mass difference $m_{u}-m_{d}$. Section 4 contains the summary and conclusions. Some more technical material is relegated to the appendices.

\section{Construction of the effective Lagrangian}

In this section, we will be concerned with the construction of the complete fourth order effective Lagrangian for two flavor baryon chiral perturbation theory. We give its relativistic and heavy baryon formulation for the reasons discussed in the introduction. Since the details of the construction are similar to the ones exposed in ref. 119, \#f we concentrate on discussing the new aspects due to the inclusion of the virtual photons. While our considerations are general, we give specific details only for the two flavor case of spontaneously broken chiral $\mathrm{SU}(2)_{L} \times \mathrm{SU}(2)_{R}$ symmetry.

\subsection{Single-nucleon effective field theory and building blocks}

The starting point of our approach is to construct the most general chiral invariant Lagrangian built from pions, nucleons and external scalar, pseudoscalar, vector, axial-vector sources and virtual photons, parametrized in terms of the vector field $A_{\mu}(x)$. The Goldstone bosons are collected in a $2 \times 2$ matrix-valued field $U(x)=u^{2}(x)$. The nucleons are described by structureless

\footnotetext{
\#4 The third order effective pion-nucleon Lagrangian was first given in another basis in 20 .
} 
relativistic spin- $\frac{1}{2}$ particles, the spinors being denoted by $\Psi(x)$ in the relativistic case or by the socalled light component $N(x)$ in the heavy fermion formulation. The effective field theory admits a low energy expansion, i.e. the corresponding effective Lagrangian can be written as

$$
\mathcal{L}_{\text {eff }}=\mathcal{L}_{\pi \pi}^{(2)}+\mathcal{L}_{\pi \pi}^{(4)}+\mathcal{L}_{\pi N}^{(1)}+\mathcal{L}_{\pi N}^{(2)}+\mathcal{L}_{\pi N}^{(3)}+\mathcal{L}_{\pi N}^{(4)}+\ldots
$$

where the ellipsis denotes terms of higher order not considered here. For the explicit form of the meson Lagrangian and the dimension one and two pion-nucleon terms, we refer to ref. 221]. More precisely, in the pion-nucleon sector, the inclusion of the virtual photons modifies the leading term of dimension one (as given below [8]) and leads to new local (contact) terms starting at second order,

$$
\mathcal{L}_{\pi N}^{(i)}=\mathcal{L}_{\pi N, \mathrm{str}}^{(i)}+\mathcal{L}_{\pi N, \mathrm{em}}^{(i)}, \quad(i=2,3,4)
$$

We are concerned here with the construction of the terms including virtual photons $\mathcal{L}_{\pi N, \mathrm{em}}^{(i)}(i=$ $2,3,4)$. The minimal form of the strong terms (no virtual photons) up-to-and-including third order can be found in ref. [19] and the divergence structure of $\mathcal{L}_{\pi N, \text { str }}^{(4)}$ is discussed in ref. [25]. Two groups are presently working on setting up the minimal fourth order strong Lagrangian [26, 27]. We remark that the various parameters appearing in the effective Lagrangian have to be taken in the chiral $\mathrm{SU}(2)$ limit $\left(m_{u}=m_{d}=0, m_{s}\right.$ fixed, $\left.e^{2}=0\right)$. The corresponding dimension two and four electromagnetic meson Lagrangians are briefly reviewed in app. A.

To construct the pertinent terms, we use the standard methods of non-linearly realized chiral symmetry. From the external sources, we construct the following building blocks starting with the nucleon covariant derivative $\nabla_{\mu}$, the chiral connection $\Gamma_{\mu}$, the chiral viel-bein $u_{\mu}$ and the external sources:

$$
\begin{aligned}
\nabla_{\mu} & =\partial_{\mu}+\Gamma_{\mu}, \\
\Gamma_{\mu} & =\frac{1}{2}\left[u^{\dagger}, \partial_{\mu} u\right]-\frac{i}{2} u^{\dagger} r_{\mu} u-\frac{i}{2} u l_{\mu} u^{\dagger} \\
u_{\mu} & =i\left(\partial_{\mu} u u^{\dagger}+u^{\dagger} \partial_{\mu} u-i u^{\dagger} r_{\mu} u+i u l_{\mu} u^{\dagger}\right) \\
r_{\mu} & =v_{\mu}+a_{\mu}, \quad l_{\mu}=v_{\mu}-a_{\mu} \\
F_{\mu \nu}^{ \pm} & =u^{\dagger} F_{\mu \nu}^{R} u \pm u F_{\mu \nu}^{L} u^{\dagger} \\
F_{\mu \nu}^{L} & =\partial_{\mu} l_{\nu}-\partial_{\nu} l_{\mu}-i\left[l_{\mu}, l_{\nu}\right] \\
F_{\mu \nu}^{R} & =\partial_{\mu} r_{\nu}-\partial_{\nu} r_{\mu}-i\left[r_{\mu}, r_{\nu}\right] \\
\chi_{ \pm} & =u^{\dagger} \chi u^{\dagger} \pm u \chi^{\dagger} u
\end{aligned}
$$

and we employ the following definition for traceless operators (' $\langle\ldots\rangle$ ' denotes the trace in flavor space)

$$
\tilde{A}=A-\frac{1}{2}\langle A\rangle
$$

Here and in what follows, $A$ denotes an arbitrary $2 \times 2$ matrix. Furthermore, $\chi(x)=s(x)+i p(x)$ includes the explicit chiral symmetry breaking through the small current quark masses, $s(x)=$ $B \operatorname{diag}\left(m_{u}, m_{d}\right)+\ldots$ and $B=|\langle 0|\bar{q} q| 0\rangle| / F_{\pi}^{2}$, with $F_{\pi}$ the (weak) pion decay constant and the 
scalar quark condensate measures the strength of the chiral symmetry breaking. We assign the following chiral dimensions to the pertinent fields and operators:

$$
\begin{aligned}
& U(x), \Psi(x), \partial_{\mu} \Psi(x), N(x)=\mathcal{O}(1), \\
& \partial_{\mu} U(x), \partial_{\mu} N(x), u_{\mu}(x), l_{\mu}(x), r_{\mu}(x)=\mathcal{O}(q), \\
& s(x), p(x), \chi_{ \pm}(x), F_{\mu \nu}^{ \pm}(x), F_{\mu \nu}^{L, R}(x)=\mathcal{O}\left(q^{2}\right),
\end{aligned}
$$

which amounts to the so-called large condensate scenario of the spontaneous chiral symmetry violation, $B \gg F_{\pi}$. Here, $q$ denotes a genuine small momentum or meson mass with respect to the typical hadronic scale of about $1 \mathrm{GeV}$.

To introduce virtual photons in the effective pion-nucleon field theory, one has to first set up to a power counting scheme for the electric charge $e$. In principle, one has to deal with two expansions, one in the small momenta/masses (the chiral expansion) and a second one in the em coupling. A priori, these two expansions can be treated separately. As proposed in ref. 2n and based on the observation that $e^{2} / 4 \pi \simeq M_{\pi}^{2} /\left(4 \pi F_{\pi}\right)^{2} \sim 1 / 100$, we count the electric charge as a small momentum,

$$
e=\mathcal{O}(q)
$$

which is the most economic way of organizing the double expansions in terms of one. In particular, since the electric charge related to the virtual photons always appears quadratic, the following pattern for the terms in the electromagnetic effective Lagrangian emerges. At second order, we can only have terms of order $e^{2}$, at third order $e^{2} q$ and at fourth order $e^{2} q^{2}$ or $e^{4}$ (besides the standard strong terms). In what follows, we will collectively denote a term of order $k$ as $\mathcal{O}\left(q^{k}\right)$ without differentiating between small momenta or em couplings. Note that the necessity of having the charge matrix appearing squared severely limits the number of possible terms. For example, em corrections to the explicit symmetry breaking operators, which are of second order in the standard counting, can therefore only appear at fourth order. The inclusion of the virtual photons proceeds as follows. In the meson sector one usually works with the quark charge matrix

$$
Q=\frac{e}{3}\left(\begin{array}{cc}
2 & 0 \\
0 & -1
\end{array}\right)=\frac{e}{6}\left(3 \tau^{3}+\mathbf{1}\right)
$$

and introduces spurions $Q_{L, R}$ with a definite transformation property under chiral $\mathrm{SU}(2)_{L} \times$ $\mathrm{SU}(2)_{R}$,

$$
Q_{I} \rightarrow g_{I} Q_{I} g_{I}^{\dagger}, \quad g_{I} \in \mathrm{SU}(2)_{I}, \quad I=L, R
$$

to construct chiral invariant building blocks. Only at the end, one sets $Q_{L}=Q_{R}=Q$ [1], 2]. In the coupled pion-nucleon sector it is natural to use the nucleon charge matrix [3, 8]

$$
Q=e\left(\begin{array}{ll}
1 & 0 \\
0 & 0
\end{array}\right)=\frac{e}{2}\left(\tau^{3}+\mathbf{1}\right),
$$

\footnotetext{
\#5 Of course, the external vector field can be a photon. These are counted in the standard manner and do not concern our arguments.
} 
and to work with the usual generalizations,

$$
Q_{ \pm}=\frac{1}{2}\left(u Q u^{\dagger} \pm u^{\dagger} Q u\right)
$$

which under chiral $\mathrm{SU}(2)_{L} \times \mathrm{SU}(2)_{R}$ symmetry transform as any matrix-valued matter field

$$
Q_{ \pm} \rightarrow K Q_{ \pm} K^{\dagger}
$$

Here, $K$ is the compensator field representing an element of the conserved subgroup $\mathrm{SU}(2)_{V}$. It depends on the pion fields in a highly non-linear fashion. Furthermore, under parity $(P)$ and charge conjugation $(C)$ transformations, one finds

$$
\begin{aligned}
& P Q_{ \pm} P^{-1}= \pm Q_{ \pm}, \\
& C Q_{ \pm} C^{-1}= \pm Q_{ \pm}^{T},
\end{aligned}
$$

where $Q^{T}$ is the transposed of the matrix $Q$. The physical results do, of course, not depend on the specific parametrization. From the decomposition

$$
Q=e\left(\alpha \mathbf{1}+\beta \tau^{3}\right)
$$

with $\alpha=1 / 6, \beta=1 / 2$ in eq.(2.14) and $\alpha=1 / 2, \beta=1 / 2$ in eq.(2.16) one derives the relation

$$
\langle Q\rangle^{2}=\frac{(2 \alpha)^{2}}{\alpha^{2}+\beta^{2}}\left\langle Q^{2}\right\rangle
$$

which allows to drop the monomials proportional to $\langle Q\rangle^{2}$ and $\langle Q\rangle^{4}$ in the Lagrangian to fourth order. In what follows we give the minimal Lagrangian to the fourth order with virtual photons. For completeness the Lagrangian of second and third order is also given. We use the notation of $\mathrm{SM}$, but prefer to work in the flavor basis with traceless objects, as in ref. [19]. Finally, we remark that throughout we work in the Landau gauge, see also app. A.

\subsection{First, second and third order terms}

In this paragraph, we briefly review what is already known about the electromagnetic effective pion-nucleon Lagrangian to third order, following closely SM (with a few differences to be discussed).

In particular, to lowest order $(i=1$ ) one finds (in the relativistic and the heavy fermion formulation)

$$
\begin{aligned}
\mathcal{L}_{\pi N}^{(1)} & =\bar{\Psi}\left(i \gamma_{\mu} \cdot \tilde{\nabla}^{\mu}-m+\frac{1}{2} g_{A} \gamma^{\mu} \gamma_{5} \cdot \tilde{u}_{\mu}\right) \Psi \\
& =\bar{N}\left(i v \cdot \tilde{\nabla}+g_{A} S \cdot \tilde{u}\right) N+\mathcal{O}\left(\frac{1}{m}\right)
\end{aligned}
$$

with

$$
\begin{aligned}
\tilde{\nabla}_{\mu} & =\nabla_{\mu}-i Q_{+} A_{\mu} \\
\tilde{u}_{\mu} & =u_{\mu}-2 Q_{-} A_{\mu}
\end{aligned}
$$


and \#9

$$
\Psi(x)=\exp \{i m v \cdot x\}(N(x)+h(x)) .
$$

Furthermore, $v_{\mu}$ denotes the nucleons' four-velocity, $S_{\mu}$ the covariant spin-vector à la PauliLubanski and $g_{A}$ the axial-vector coupling constant. We also need the generalized pion covariant derivative containing the external vector $\left(v_{\mu}\right)$ and axial-vector $\left(a_{\mu}\right)$ sources,

$$
\mathrm{d}_{\mu} U=\partial_{\mu} U-i\left(v_{\mu}+a_{\mu}+Q A_{\mu}\right) U+i U\left(v_{\mu}-a_{\mu}+Q A_{\mu}\right) .
$$

These virtual photon effects can only come in via loop diagrams since by definition a virtual photon can not be an asymptotic state. Note that soft photon radiation can be treated by standard methods and will not be discussed any further.

At second order $(i=2)$, local contact terms with finite low-energy constants (LECs) appear. Following SM, we call these LECs $f_{i}$ for the heavy baryon approach and $f_{i}^{\prime}$ in the relativistic Lagrangian. As stated before, the em Lagrangian is given entirely in terms of squares of $Q_{ \pm}$(and their traceless companions),

$$
\mathcal{L}_{\pi N, \mathrm{em}}^{(2)}=\sum_{i=1}^{3} F_{\pi}^{2} f_{i}^{\prime} \bar{\Psi} \mathcal{O}_{i}^{(2)} \Psi=\sum_{i=1}^{3} F_{\pi}^{2} f_{i} \bar{N} \mathcal{O}_{i}^{(2)} N,
$$

with the $\mathcal{O}_{i}^{(2)}$ monomials of dimension two,

$$
\mathcal{O}_{1}^{(2)}=\left\langle\tilde{Q}_{+}^{2}-\tilde{Q}_{-}^{2}\right\rangle, \quad \mathcal{O}_{2}^{(2)}=\left\langle Q_{+}\right\rangle \tilde{Q}_{+}, \quad \mathcal{O}_{3}^{(2)}=\left\langle\tilde{Q}_{+}^{2}+\tilde{Q}_{-}^{2}\right\rangle .
$$

Note that we have one term less than given in SM. The third and fourth term in SM both lead to an overall (unobservable) em shift $e^{2} \bar{\Psi} \Psi$ or $e^{2} \bar{N} N$ of the nucleon mass in the chiral limit and thus can be lumped in one term. Notice furthermore that only the second term in eq.(2.28) has an isovector piece and contributes to the neutron-proton mass difference [8]. The factor $F_{\pi}^{2}$ in eq.(2.28) ensures that the em LECs have the same dimension as the corresponding strong dimension two LECs. From the third order calculation of the proton-neutron mass difference [8] one deduces the value for $f_{2}, f_{2}=-(0.45 \pm 0.19) \mathrm{GeV}^{-1}$.

The Lagrangian to third order takes the form

$$
\mathcal{L}_{\pi N, \mathrm{em}}^{(3)}=\sum_{i=1}^{12} F_{\pi}^{2} g_{i}^{\prime} \bar{\Psi} \mathcal{O}_{i}^{(3)} \Psi=\sum_{i=1}^{12} F_{\pi}^{2} g_{i} \bar{N} \mathcal{O}_{i}^{(3)} N
$$

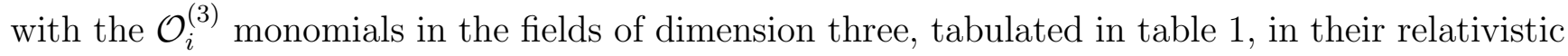
form and the heavy baryon counterparts. Again, for the $g_{i}$ to have the same mass dimension as the $d_{i}$ of the strong sector defined in ref. [19], we have multiplied them with a factor of $F_{\pi}^{2}$. Thus the $g_{i}\left(g_{i}^{\prime}\right)$ scales as $\left[\right.$ mass $\left.^{-2}\right]$.

Note that we have fewer terms than given in ref. [8]. First, we have used the nucleon equations of motion and also dropped all terms with a single $\left\langle Q_{+}\right\rangle^{2}$ since these can be lumped together with

\footnotetext{
${ }^{\# 6}$ We do not spell out the details of how to construct the heavy nucleon EFT from its relativistic counterpart but refer the reader to the extensive review [21].
} 


\begin{tabular}{|r|c||c|c|}
\hline i & Relat. & HBCHPT & $\kappa_{i}$ \\
\hline 1 & $\gamma^{\mu} \gamma_{5}<\tilde{Q}_{+} u_{\mu}>\tilde{Q}_{+}$ & $<\tilde{Q}_{+} S \cdot u>\tilde{Q}_{+}$ & $4 Z g_{A}\left(1-g_{A}^{2}\right)$ \\
2 & $\gamma^{\mu} \gamma_{5}<\tilde{Q}_{-} u_{\mu}>\tilde{Q}_{-}$ & $<\tilde{Q}_{-} S \cdot u>\tilde{Q}_{-}$ & $2 g_{A}\left(1-g_{A}^{2}\right)(1-2 Z)$ \\
3 & $\gamma^{\mu} \gamma_{5}<\tilde{Q}_{+} u_{\mu}>Q_{+}>$ & $<\tilde{Q}_{+} S \cdot u><Q_{+}>$ & $-2 g_{A}$ \\
4 & $\gamma^{\mu} \gamma_{5}<\tilde{Q}_{+}^{2}+\tilde{Q}_{-}^{2}>u_{\mu}$ & $<\tilde{Q}_{+}^{2}+\tilde{Q}_{-}^{2}>S \cdot u$ & $-g_{A}\left(-4-g_{A}^{2}\right) / 2$ \\
5 & $\gamma^{\mu} \gamma_{5}<\tilde{Q}_{+}^{2}-\tilde{Q}_{-}^{2}>u_{\mu}$ & $<\tilde{Q}_{+}^{2}-\tilde{Q}_{-}^{2}>S \cdot u$ & $g_{A}\left(8 Z-g_{A}^{2}\right) / 2$ \\
6 & $\gamma^{\mu}<\tilde{Q}_{+} u_{\mu}>\tilde{Q}_{-}$ & $<\tilde{Q}_{+} v \cdot u>\tilde{Q}_{-}$ & -4 \\
7 & $\gamma^{\mu}<\tilde{Q}_{-} u_{\mu}>\tilde{Q}_{+}$ & $<\tilde{Q}_{-} v \cdot u>\tilde{Q}_{+}$ & $1-3 g_{A}^{2}$ \\
8 & $\gamma^{\mu}<\tilde{Q}_{-} u_{\mu}><Q_{+}>$ & $<\tilde{Q}_{-} v \cdot u><Q_{+}>$ & 0 \\
9 & $\gamma^{\mu} \gamma_{5}\left[\tilde{Q}_{+},\left[i \nabla_{\mu}, \tilde{Q}_{+}\right]\right]$ & {$\left[\tilde{Q}_{+},\left[i S \cdot \nabla, \tilde{Q}_{+}\right]\right]$} & -2 \\
10 & $\gamma^{\mu} \gamma_{5}\left[\tilde{Q}_{-},\left[i \nabla_{\mu}, \tilde{Q}_{-}\right]\right]$ & {$\left[\tilde{Q}_{-},\left[i S \cdot \nabla, \tilde{Q}_{-}\right]\right]$} & $\left(1-9 g_{A}^{2}\right) / 2$ \\
11 & $\gamma^{\mu}\left[\tilde{Q}_{+},\left[i \nabla_{\mu}, \tilde{Q}_{-}\right]\right]$ & {$\left[\tilde{Q}_{+},\left[i v \cdot \nabla, \tilde{Q}_{-}\right]\right]$} & 0 \\
12 & $\gamma^{\mu}\left[\tilde{Q}_{-},\left[i \nabla_{\mu}, \tilde{Q}_{+}\right]\right]$ & {$\left[\tilde{Q}_{-},\left[i v \cdot \nabla, \tilde{Q}_{+}\right]\right]$} & 0 \\
\hline
\end{tabular}

Table 1: Monomials $\mathcal{O}_{i}$ of third order contributing to the em Lagrangian for the relativistic and heavy baryon formulations. For HBCHPT, the $\beta$-functions $\kappa_{i}$ are also given. Here, $Z=C / F_{\pi}^{4}$.

the similar terms $\sim\left\langle\tilde{Q}_{+}^{2}+\tilde{Q}_{-}^{2}\right\rangle$. For example, the terms 4 and 6 of table 1 in [ 8$]$ both lead to an electromagnetic renormalization of the axial-vector coupling constant and can thus effectively be represented by one term. The low-energy constants $g_{i}^{\prime}\left(g_{i}\right)$ absorb the divergences in the standard manner (given here for HBCHPT),

$$
\begin{aligned}
g_{i} & =F_{\pi}^{2} \kappa_{i} L+g_{i}^{r}(\mu) \\
L & =\frac{\mu^{d-4}}{16 \pi^{2}}\left\{\frac{1}{d-4}-\frac{1}{2}\left[\ln (4 \pi)+\Gamma^{\prime}(1)+1\right]\right\}
\end{aligned}
$$

with $\mu$ the scale of dimensional regularization and $d$ the number of space-time dimensions. The $g_{i}^{r}(\mu)$ are the renormalized, finite, scale-dependent and dimensionless low-energy constants. These can be fixed by data or have to be estimated with the help of some model. They obey the standard renormalization group equation. The $\beta$-functions $\kappa_{i}$ given in table 1 agree with the ones of [8] after appropriate rearrangement. In addition to these terms, there are three which are only needed for renormalization, i.e. in HBCHPT they are proportional to $\omega=v \cdot l$, with $l$ the small residual nucleon momentum. The corresponding monomials and their $\beta$-functions read

$$
\begin{array}{lll}
\mathcal{O}_{13}^{(3)}=\left\langle\tilde{Q}_{+}^{2}-\tilde{Q}_{-}^{2}\right\rangle i v \cdot \nabla+\text { h.c. }, & \kappa_{13}=-1 / 2-3 g_{A}^{2}(1+8 Z) / 4 \\
\mathcal{O}_{14}^{(3)}=\left\langle\tilde{Q}_{+}^{2}+\tilde{Q}_{-}^{2}\right\rangle i v \cdot \nabla+\text { h.c. }, & \kappa_{14}=-1 / 2+3 g_{A}^{2} / 4 \\
\mathcal{O}_{15}^{(3)}=\left\langle Q_{+}\right\rangle \tilde{Q}_{+} i v \cdot \nabla+\text { h.c. }, & & \kappa_{15}=-2 .
\end{array}
$$

In the relativistic formulation, these terms would formally count as second order. Note also that one could use the following relations to transfrom some of the terms in table 1, but this is equivalent 
to a redefinition of a few counterterms and does not reduce the number of terms [8],

$$
\begin{aligned}
{\left[\nabla_{\mu}, Q_{ \pm}\right] } & =-\frac{i}{2}\left[u_{\mu}, Q_{\mp}\right]+c_{\mu}^{ \pm} \\
c_{\mu}^{ \pm} & =\frac{1}{2}\left\{u\left(\partial_{\mu} Q-i\left[v_{\mu}-a_{\mu}, Q\right]\right) u^{\dagger} \pm u^{\dagger}\left(\partial_{\mu} Q-i\left[v_{\mu}+a_{\mu}, Q\right]\right) u\right\} \\
{\left[\nabla^{\mu}, u_{\mu}\right] } & =\frac{i}{2} \chi_{-}-\frac{i}{4}\left\langle\chi_{-}\right\rangle+i \frac{4 C}{F^{2}}\left[Q_{+}, Q_{-}\right]+\mathcal{O}\left(q^{4}\right) .
\end{aligned}
$$

where the LEC $C$ is defined in app. A.

\subsection{Fourth order terms}

\begin{tabular}{|r|c|c|}
\hline $\mathrm{i}$ & Relat. & HBCHPT \\
\hline 12 & $\left\langle\tilde{Q}_{+}^{2}+\tilde{Q}_{-}^{2}\right\rangle\left\langle u_{\mu} u_{\nu}\right\rangle\left\{\nabla^{\mu}, \nabla^{\nu}\right\}+$ h.c. & $\left\langle\tilde{Q}_{+}^{2}+\tilde{Q}_{-}^{2}\right\rangle\left\langle(v \cdot u)^{2}\right\rangle$ \\
13 & $\left\langle\tilde{Q}_{+}^{2}-\tilde{Q}_{-}^{2}\right\rangle\left\langle u_{\mu} u_{\nu}\right\rangle\left\{\nabla^{\mu}, \nabla^{\nu}\right\}+$ h.c. & $\left\langle\tilde{Q}_{+}^{2}-\tilde{Q}_{-}^{2}\right\rangle\left\langle(v \cdot u)^{2}\right\rangle$ \\
14 & $\left\langle\tilde{Q}_{+} u_{\mu}\right\rangle\left\langle\tilde{Q}_{+} u_{\nu}\right\rangle\left\{\nabla^{\mu}, \nabla^{\nu}\right\}+$ h.c. & $\left\langle\tilde{Q}_{+} v \cdot u\right\rangle\left\langle\tilde{Q}_{+} v \cdot u\right\rangle$ \\
15 & $\left\langle\tilde{Q}_{-} u_{\mu}\right\rangle\left\langle\tilde{Q}_{-} u_{\nu}\right\rangle\left\{\nabla^{\mu}, \nabla^{\nu}\right\}+$ h.c. & $\left\langle\tilde{Q}_{-} v \cdot u\right\rangle\left\langle\tilde{Q}_{-} v \cdot u\right\rangle$ \\
16 & $\left\langle Q_{+}\right\rangle\left\langle u_{\mu} u_{\nu}\right\rangle \tilde{Q}_{+}\left\{\nabla^{\mu}, \nabla^{\nu}\right\}+$ h.c. & $\left\langle Q_{+}\right\rangle\left\langle(v \cdot u)^{2}\right\rangle \tilde{Q}_{+}$ \\
17 & $\left\langle Q_{+}\right\rangle\left\langle\tilde{Q}_{+} u_{\mu}\right\rangle u_{\nu}\left\{\nabla^{\mu}, \nabla^{\nu}\right\}+$ h.c. & $\left\langle Q_{+}\right\rangle\left\langle\tilde{Q}_{+} v \cdot u\right\rangle v \cdot u$ \\
\hline 18 & $i \sigma^{\mu \nu}\left\langle\tilde{Q}_{+}\left[u_{\mu}, u_{\nu}\right]\right\rangle \tilde{Q}_{+}$ & $v_{\rho} \varepsilon^{\rho \mu \nu \sigma} S_{\sigma}\left\langle\tilde{Q}_{+}\left[u_{\mu}, u_{\nu}\right]\right\rangle \tilde{Q}_{+}$ \\
19 & $i \sigma^{\mu \nu}\left\langle\tilde{Q}_{-}\left[u_{\mu}, u_{\nu}\right]\right\rangle \tilde{Q}_{-}$ & $v_{\rho} \varepsilon^{\rho \mu \nu \sigma} S_{\sigma}\left\langle\tilde{Q}_{-}\left[u_{\mu}, u_{\nu}\right]\right\rangle \tilde{Q}_{-}$ \\
20 & $i \sigma^{\mu \nu}\left\langle\tilde{Q}_{+}^{2}+\tilde{Q}_{-}^{2}\right\rangle\left[u_{\mu}, u_{\nu}\right]$ & $v_{\rho} \varepsilon^{\rho \mu \nu \sigma} S_{\sigma}\left\langle\tilde{Q}_{+}^{2}+\tilde{Q}_{-}^{2}\right\rangle\left[u_{\mu}, u_{\nu}\right]$ \\
21 & $i \sigma^{\mu \nu}\left\langle\tilde{Q}_{+}^{2}-\tilde{Q}_{-}^{2}\right\rangle\left[u_{\mu}, u_{\nu}\right]$ & $v_{\rho} \varepsilon^{\rho \mu \nu \sigma} S_{\sigma}\left\langle\tilde{Q}_{+}^{2}-\tilde{Q}_{-}^{2}\right\rangle\left[u_{\mu}, u_{\nu}\right]$ \\
22 & $i \sigma^{\mu \nu}\left\langle\tilde{Q}_{+} u_{\mu}\right\rangle\left[\tilde{Q}_{+}, u_{\nu}\right]$ & $v_{\rho} \varepsilon^{\rho \mu \nu \sigma} S_{\sigma}\left\langle\tilde{Q}_{+} u_{\mu}\right\rangle\left[\tilde{Q}_{+}, u_{\nu}\right]$ \\
23 & $i \sigma^{\mu \nu}\left\langle\tilde{Q}_{-} u_{\mu}\right\rangle\left[\tilde{Q}_{-}, u_{\nu}\right]$ & $v_{\rho} \varepsilon^{\rho \mu \nu \sigma} S_{\sigma}\left\langle\tilde{Q}_{-} u_{\mu}\right\rangle\left[\tilde{Q}_{-}, u_{\nu}\right]$ \\
24 & $i \sigma^{\mu \nu}\left\langle Q_{+}\right\rangle\left\langle\tilde{Q}_{+}\left[u_{\mu}, u_{\nu}\right]\right\rangle$ & $v_{\rho} \varepsilon^{\rho \mu \nu \sigma} S_{\sigma}\left\langle Q_{+}\right\rangle\left\langle\tilde{Q}_{+}\left[u_{\mu}, u_{\nu}\right]\right\rangle$ \\
\hline 25 & $\gamma^{\mu} \gamma_{5}\left\langle\tilde{Q}_{+} u_{\mu}\right\rangle\left\langle\tilde{Q}_{-} u_{\nu}\right\rangle i \nabla^{\nu}+$ h.c. & $i\left\langle\tilde{Q}_{+} S \cdot u\right\rangle\left\langle\tilde{Q}_{-} v \cdot u\right\rangle$ \\
26 & $\gamma^{\nu} \gamma_{5}\left\langle\tilde{Q}_{+} u_{\mu}\right\rangle\left\langle\tilde{Q}_{-} u_{\nu}\right\rangle i \nabla^{\mu}+$ h.c. & $i\left\langle\tilde{Q}_{+} v \cdot u\right\rangle\left\langle\tilde{Q}_{-} S \cdot u\right\rangle$ \\
27 & $\gamma^{\mu} \gamma_{5}\left\langle Q_{+}\right\rangle\left\langle\tilde{Q}_{-} u_{\mu}\right\rangle u_{\nu} i \nabla^{\nu}+$ h.c. & $i\left\langle Q_{+}\right\rangle\left\langle\tilde{Q}_{-} S \cdot u\right\rangle v \cdot u$ \\
28 & $\gamma^{\nu} \gamma_{5}\left\langle Q_{+}\right\rangle\left\langle\tilde{Q}_{-} u_{\mu}\right\rangle u_{\nu} i \nabla^{\mu}+$ h.c. & $i\left\langle Q_{+}\right\rangle\left\langle\tilde{Q}_{-} v \cdot u\right\rangle S \cdot u$ \\
\hline
\end{tabular}

Table 2: "Pion" terms of fourth order. The terms 18-24 (25-28) involve at least two (three) pions.

We now consider the terms of fourth order. We split these into various classes according to the number and type of external fields. In the absence of any external fields, we have the so-called "pion" terms. Alltogether, there are 28 such terms from which 11 have the same structure in the relativistic and the HBCHPT formulations. The corresponding monomials for these particular terms read

$$
\mathcal{O}_{1}^{(4)}=\left\langle\tilde{Q}_{+}^{2}+\tilde{Q}_{-}^{2}\right\rangle^{2}, \quad \mathcal{O}_{2}^{(4)}=\left\langle\tilde{Q}_{+}^{2}-\tilde{Q}_{-}^{2}\right\rangle^{2}, \quad \mathcal{O}_{3}^{(4)}=\left\langle\tilde{Q}_{+}^{2}+\tilde{Q}_{-}^{2}\right\rangle\left\langle\tilde{Q}_{+}^{2}-\tilde{Q}_{-}^{2}\right\rangle,
$$




$$
\begin{aligned}
& \mathcal{O}_{4}^{(4)}=\left\langle\tilde{Q}_{+}^{2}+\tilde{Q}_{-}^{2}\right\rangle\left\langle Q_{+}\right\rangle \tilde{Q}_{+}, \quad \mathcal{O}_{5}^{(4)}=\left\langle\tilde{Q}_{+}^{2}-\tilde{Q}_{-}^{2}\right\rangle\left\langle Q_{+}\right\rangle \tilde{Q}_{+}, \quad \mathcal{O}_{6}^{(4)}=\left\langle\tilde{Q}_{+}^{2}+\tilde{Q}_{-}^{2}\right\rangle\left\langle u^{2}\right\rangle \\
& \mathcal{O}_{7}^{(4)}=\left\langle\tilde{Q}_{+}^{2}-\tilde{Q}_{-}^{2}\right\rangle\left\langle u^{2}\right\rangle, \quad \mathcal{O}_{8}^{(4)}=\left\langle\tilde{Q}_{+} u_{\mu}\right\rangle\left\langle\tilde{Q}_{+} u^{\mu}\right\rangle, \quad \mathcal{O}_{9}^{(4)}=\left\langle\tilde{Q}_{-} u_{\mu}\right\rangle\left\langle\tilde{Q}_{-} u^{\mu}\right\rangle \\
& \mathcal{O}_{10}^{(4)}=\left\langle Q_{+}\right\rangle\left\langle u^{2}\right\rangle \tilde{Q}_{+}, \quad \mathcal{O}_{11}^{(4)}=\left\langle Q_{+}\right\rangle\left\langle\tilde{Q}_{+} u_{\mu}\right\rangle u^{\mu}
\end{aligned}
$$

From these, $\mathcal{O}_{4,5,10}$ are proportional to $\tau^{3}$, i.e. contribute differently to reactions involving a proton and a neutron. Note also that the first five monomials are all $\mathcal{O}\left(e^{4}\right)$, they thus involve no small external momentum or meson mass. While the first term is a pure em shift to the nucelon mass, the other four operators can contribute to pion-nucleon scattering through their $\bar{\Psi} \Psi \pi \pi / \bar{N} N \pi \pi-$ vertices. The other terms are given in table 2. We remark that the "pion" terms involving the covariant derivatives also contain contributions from external fields. Of particular interest are the terms which include explicit chiral symmetry breaking through the external fields $\chi_{+}$and $\tilde{\chi}_{+}$. Note that $\tilde{\chi}_{+}=0$ for $m_{u}=m_{d}$. There are six such terms, which obviously have the same structure in the relativistic and the heavy fermion framework. These read:

$$
\begin{aligned}
& \mathcal{O}_{29}^{(4)}=\left\langle\tilde{Q}_{+}^{2}+\tilde{Q}_{-}^{2}\right\rangle \tilde{\chi}_{+}, \quad \mathcal{O}_{30}^{(4)}=\left\langle\tilde{Q}_{+}^{2}-\tilde{Q}_{-}^{2}\right\rangle \tilde{\chi}_{+}, \quad \mathcal{O}_{31}^{(4)}=\left\langle Q_{+}\right\rangle\left\langle\tilde{Q}_{+} \tilde{\chi}_{+}\right\rangle, \\
& \mathcal{O}_{32}^{(4)}=\left\langle\tilde{Q}_{+}^{2}+\tilde{Q}_{-}^{2}\right\rangle\left\langle\chi_{+}\right\rangle, \quad \mathcal{O}_{33}^{(4)}=\left\langle\tilde{Q}_{+}^{2}-\tilde{Q}_{-}^{2}\right\rangle\left\langle\chi_{+}\right\rangle, \quad \mathcal{O}_{34}^{(4)}=\left\langle Q_{+}\right\rangle \tilde{Q}_{+}\left\langle\chi_{+}\right\rangle .
\end{aligned}
$$

Again, only three of these terms are proportional to $\tau^{3}$, these are $\mathcal{O}_{29,30,34}^{(4)}$. The first three of these are $\sim e^{2}\left(m_{u}-m_{d}\right)$ while the others are $\sim e^{2}\left(m_{u}+m_{d}\right)$. In addition, we find three terms which to leading order are proportional to external pseudoscalar sources $\left(\chi_{-}, \tilde{\chi}_{-}\right)$,

$$
\mathcal{O}_{35}^{(4)}=\left\langle Q_{+}\right\rangle\left[i \tilde{Q}_{-}, \tilde{\chi}_{-}\right], \quad \mathcal{O}_{36}^{(4)}=\left\langle\left[\tilde{Q}_{+}, \tilde{Q}_{-}\right] \tilde{\chi}_{-}\right\rangle, \quad \mathcal{O}_{37}^{(4)}=\left[\tilde{Q}_{+}, \tilde{Q}_{-}\right]\left\langle\chi_{-}\right\rangle
$$

The terms proportional to the field strength tensor $F_{\mu \nu}^{ \pm}$build from the external vector and axialvector fields are tabulated in table 3. These are relevant for the virtual photon contributions to the magnetic moments, em and strange nucleon form factors or in pion photo/electroproduction off nucleons.

In total, we have enumerated 50 terms so far. There are another 40 terms, listed in app. Q. These are, however, of no direct relevance since by use of the equations of motion they can all be transformed into structures $\sim c_{\mu}^{ \pm}$, cf. eq.(2.34), and are thus only needed for processes with external axial-vector fields or low-energy manifestations of $Z^{0}$-exchange. Such a procedure would, of course, also lead to some terms already present in the list discussed so far, i.e. the corresponding LECs would have to be readjusted. So the complete and minimal fourth order pion-nucleon Lagrangian with virtual photons is given by

$$
\begin{aligned}
\mathcal{L}_{\pi N, \mathrm{em}}^{(4)} & =\sum_{i=1}^{5} F_{\pi}^{4} h_{i}^{\prime} \bar{\Psi} \mathcal{O}_{i}^{\left(e^{4}\right)} \Psi+\sum_{i=6}^{90} F_{\pi}^{2} h_{i}^{\prime} \bar{\Psi} \mathcal{O}_{i}^{\left(e^{2} p^{2}\right)} \Psi \\
& =\sum_{i=1}^{5} F_{\pi}^{4} h_{i} \bar{\Psi} \mathcal{O}_{i}^{\left(e^{4}\right)} \Psi+\sum_{i=6}^{90} F_{\pi}^{2} h_{i} \bar{\Psi} \mathcal{O}_{i}^{\left(e^{2} p^{2}\right)} \Psi
\end{aligned}
$$

with the $\mathcal{O}_{i}^{(4)}$ monomials in the fields of dimension four, given in this paragraph and in app. Q. To be consistent with the scaling properties of the dimension two and three LECs, the $h_{i}$ are multiplied with powers of $F_{\pi}^{2}$ such that the first five LECs take dimension [mass ${ }^{-3}$ ] while the others are of 


\begin{tabular}{|r|c|c|}
\hline $\mathrm{k}$ & Relat. & HBCHPT \\
\hline 38 & $\sigma^{\mu \nu}\left\langle\tilde{Q}_{+}^{2}+\tilde{Q}_{-}^{2}\right\rangle \tilde{F}_{\mu \nu}^{+}$ & $v_{\rho} \varepsilon^{\rho \mu \nu \sigma} S_{\sigma}\left\langle\tilde{Q}_{+}^{2}+\tilde{Q}_{-}^{2}\right\rangle \tilde{F}_{\mu \nu}^{+}$ \\
39 & $\sigma^{\mu \nu}\left\langle\tilde{Q}_{+}^{2}-\tilde{Q}_{-}^{2}\right\rangle \tilde{F}_{\mu \nu}^{+}$ & $v_{\rho} \varepsilon^{\rho \mu \nu \sigma} S_{\sigma}\left\langle\tilde{Q}_{+}^{2}-\tilde{Q}_{-}^{2}\right\rangle \tilde{F}_{\mu \nu}^{+}$ \\
40 & $\sigma^{\mu \nu}\left\langle Q_{+}\right\rangle\left\langle\tilde{Q}_{+} \tilde{F}_{\mu \nu}^{+}\right\rangle$ & $v_{\rho} \varepsilon^{\rho \mu \nu \sigma} S_{\sigma}\left\langle Q_{+}\right\rangle\left\langle\tilde{Q}_{+} \tilde{F}_{\mu \nu}^{+}\right\rangle$ \\
41 & $\sigma^{\mu \nu}\left\langle\tilde{Q}_{+}^{2}+\tilde{Q}_{-}^{2}\right\rangle\left\langle F_{\mu \nu}^{+}\right\rangle$ & $v_{\rho} \varepsilon^{\rho \mu \nu \sigma} S_{\sigma}\left\langle\tilde{Q}_{+}^{2}+\tilde{Q}_{-}^{2}\right\rangle\left\langle F_{\mu \nu}^{+}\right\rangle$ \\
42 & $\sigma^{\mu \nu}\left\langle\tilde{Q}_{+}^{2}-\tilde{Q}_{-}^{2}\right\rangle\left\langle F_{\mu \nu}^{+}\right\rangle$ & $v_{\rho} \varepsilon^{\rho \mu \nu \sigma} S_{\sigma}\left\langle\tilde{Q}_{+}^{2}-\tilde{Q}_{-}^{2}\right\rangle\left\langle F_{\mu \nu}^{+}\right\rangle$ \\
43 & $\sigma^{\mu \nu}\left\langle Q_{+}\right\rangle \tilde{Q}_{+}\left\langle F_{\mu \nu}^{+}\right\rangle$ & $v_{\rho} \varepsilon^{\rho \mu \nu \sigma} S_{\sigma}\left\langle Q_{+}\right\rangle \tilde{Q}_{+}\left\langle F_{\mu \nu}^{+}\right\rangle$ \\
44 & $\sigma^{\mu \nu} \gamma_{5}\left\langle\left[\tilde{Q}_{+}, \tilde{Q}_{-}\right] \tilde{F}_{\mu \nu}^{+}\right\rangle$ & $i v^{\mu} S^{\nu}\left\langle\left[\tilde{Q}_{+}, \tilde{Q}_{-}\right] \tilde{F}_{\mu \nu}^{+}\right\rangle$ \\
45 & $\sigma^{\mu \nu} \gamma_{5}\left[\tilde{Q}_{+}, \tilde{Q}_{-}\right]\left\langle F_{\mu \nu}^{+}\right\rangle$ & $i v^{\mu} S^{\nu}\left[\tilde{Q}_{+}, \tilde{Q}_{-}\right]\left\langle F_{\mu \nu}^{+}\right\rangle$ \\
46 & $\sigma^{\mu \nu} \gamma_{5}\left\langle Q_{+}\right\rangle\left[\tilde{Q}_{-}, \tilde{F}_{\mu \nu}^{+}\right]$ & $i v^{\mu} S^{\nu}\left\langle Q_{+}\right\rangle\left[\tilde{Q}_{-}, \tilde{F}_{\mu \nu}^{+}\right]$ \\
\hline 47 & $\sigma^{\mu \nu}\left\langle Q_{+}\right\rangle\left\langle\tilde{Q}_{-} \tilde{F}_{\mu \nu}^{-}\right\rangle$ & $v_{\rho} \varepsilon^{\rho \mu \nu \sigma} S_{\sigma}\left\langle Q_{+}\right\rangle\left\langle\tilde{Q}_{-} \tilde{F}_{\mu \nu}^{-}\right\rangle$ \\
48 & $\sigma^{\mu \nu} \tilde{Q}_{+}\left\langle\tilde{Q}_{-} \tilde{F}_{\mu \nu}^{-}\right\rangle$ & $v_{\rho} \varepsilon^{\rho \mu \nu \sigma} S_{\sigma} \tilde{Q}_{+}\left\langle\tilde{Q}_{-} \tilde{F}_{\mu \nu}^{-}\right\rangle$ \\
49 & $\sigma^{\mu \nu} \tilde{Q}_{-}\left\langle\tilde{Q}_{+} \tilde{F}_{\mu \nu}^{-}\right\rangle$ & $v_{\rho} \varepsilon^{\rho \mu \nu \sigma} S_{\sigma} \tilde{Q}_{-}\left\langle\tilde{Q}_{+} \tilde{F}_{\mu \nu}^{-}\right\rangle$ \\
50 & $\sigma^{\mu \nu} \gamma_{5}\left\langle Q_{+}\right\rangle\left[\tilde{Q}_{+}, \tilde{F}_{\mu \nu}^{-}\right]$ & $i v^{\mu} S^{\nu}\left\langle Q_{+}\right\rangle\left[\tilde{Q}_{+}, \tilde{F}_{\mu \nu}^{-}\right]$ \\
\hline
\end{tabular}

Table 3: Fourth order terms with one external photon (38-46) and one external axial source $(47-50)$.

dimension $\left[\right.$ mass $\left.^{-1}\right]$. The corresponding $\beta$-functions are defined in analogy to eq.(2.31) and are called $\delta_{i}$,

$$
h_{i}=h_{i}^{r}(\lambda)+F_{\pi}^{2} \delta_{i} L .
$$

Note that the explicit factor of $F_{\pi}^{2}$ in front of the $\delta_{i}$ appearing in eq.(2.40) is due to our convention for the LECs used in eq.(2.39). Furthermore, the strong fourth-order $\beta$-functions determined in ref. 225] are also called $\delta_{i}$. However, due to the different sturcture of the strong and em operators at fourth order, no confusion can arise. Before applying this EFT to some physical processes, we briefly discuss the numerical values of the em LECs.

\subsection{Dimensional analysis of the LECs}

Since very little empirical information exists to pin down the em LECs $f_{i}, g_{i}$ and $h_{i}$ appearing at second, third and fourth order, we have to resort to dimensional analysis to get some idea about their values. We had already argued that each power of a charge matrix $Q$ appearing in any monomial is accompanied by a factor of $F_{\pi}$ so that the corresponding LECs have the same mass dimension as their strong (IC) counterparts. Therefore, the $f_{i}, h_{6 \ldots 90}, g_{i}$ and $h_{1 \ldots 5}$ scale as $\left[\mathrm{mass}^{-1}\right]$, $\left[\right.$ mass $\left.^{-1}\right],\left[\mathrm{mass}^{-2}\right]$ and $\left[\mathrm{mass}^{-3}\right]$, in order. Furthermore, the factors of $F_{\pi}$ are proportional to the natural low energy scale, $\left\langle 0\left|A_{\mu}^{a}(0)\right| \pi^{b}(x)\right\rangle=e^{i p x} p_{\mu} \delta^{a b} F_{\pi}$. The non-vanishing of the pion decay constant in the chiral limit is a necessary and sufficient condition for spontaneous chiral symmetry breaking. The physical origin of the em LECs is the integration of hard photon loops. Therefore, each power in $e^{2}$, as it is the case in QED, is really a power in the fine structure constant $\alpha=e^{2} / 4 \pi$. 
Thus, since the natural scale of chiral symmetry breaking is $\Lambda_{\chi} \sim m_{N} \sim M_{\rho} \sim 1 \mathrm{GeV}$, we can deduce the following estimates on the renormalized em LECs at the typical hadronic scale which we chose as $M_{\rho}$ (naturalness conditions)

$$
f_{i}=\frac{\tilde{f}_{i}}{4 \pi}, \quad g_{i}^{r}\left(M_{\rho}\right)=\frac{\tilde{g}_{i}}{4 \pi}, \quad h_{1 \ldots 5}^{r}\left(M_{\rho}\right)=\frac{\tilde{h}_{1 \ldots .5}}{(4 \pi)^{2}}, \quad h_{6 \ldots 90}^{r}\left(M_{\rho}\right)=\frac{\tilde{h}_{6 \ldots 90}}{4 \pi},
$$

with the $\tilde{f}_{i}, \tilde{g}_{i}$ and $\tilde{h}_{i}$ are numbers of order one,

$$
\tilde{f}_{i} \sim \tilde{g}_{i} \sim \tilde{h}_{i}=\mathcal{O}(1) .
$$

The $f_{i}$ are finite and scale-independent since loops only start at third order. Of course, such type of analysis does not allow to fix the signs of the LECs. For the estimates given in the next section, we choose these always to be positive. One should also remember that the numbers of order one appearing in eq.(2.41) can be sizeably smaller or larger than one. From the determination of $f_{2}$ from the the neutron-proton mass difference to third order in MS we conclude e.g. that $\tilde{f}_{2}=-5.65$. It would be interesting to develop some model which would allow one to calculate or estimate these LECs (for an attempt in the meson sector, we refer to ref. [29]).

\section{Applications}

In this section, we work out the virtual photon effects for various observables. Although we gave the effective Lagrangian in the relativistic as well as in the heavy fermion framework, we will restrict ourselves from here on to HBCHPT. We write the nucleon four-momentum $p_{\mu}$ as $p_{\mu}=m v_{\mu}+l_{\mu}$ and consider small residual momenta, $v \cdot l \ll m$. For more details on the approach we refer to the review [21]. A note on our nomenclature is necessary. In what follows, we will denote the strong isospin-conversing contributions simply as "strong" and em stands for all isospin-violating terms, may they be of pure photonic $\left(\sim e^{2 n}\right)$, pure strong $\left(\sim\left(m_{u}-m_{d}\right)^{m}\right)$ or mixed $\sim\left(e^{2 k}\left(m_{u}-m_{d}\right)^{p}\right)$ origin (with $k, m, n, p$ integer). This has to be kept in mind in the following.

\subsection{Nucleon self-energy and mass shift}

Denoting by $\omega=v \cdot l$ the off-shellness of the nucleon, the one-loop third order em contribution to the nucleon self-energy can be readily evaluated from the two graphs shown in fig. 1. In addition, one has to calculate the standard strong contribution (see e.g. [13, 28]). However, as was already argued in [0], the natural reference mass in the absence of em effects is the neutral pion mass, since the pion mass difference is almost entirely of em origin. Therefore, we express the strong contribution in terms of the neutral pion mass. In addition, one has to consider the photon loop graph. To handle the IR divergences related to the zero photon mass, we introduce a small photon mass $m_{\gamma}$ which leads to the modified photon propagator,

$$
D_{\mu \nu}(x)=\int \frac{d^{d} k}{(2 \pi)^{d}} \frac{i g_{\mu \nu}}{k^{2}-m_{\gamma}^{2}+i \epsilon} .
$$


Observables are, of course, independent of $m_{\gamma}$. Putting pieces together, we find for the self-energy to $\mathcal{O}\left(q^{3}\right)$ after performing the standard CHPT renormalization,

$$
\begin{aligned}
\Sigma^{(3, \mathrm{str}+\mathrm{em})}(\omega) & =\Sigma_{\text {loop }}^{(3, \mathrm{str}+\mathrm{em})}(\omega)+\Sigma_{\mathrm{div}}^{(3, \mathrm{str}+\mathrm{em})}(\omega), \\
\Sigma^{(3, \mathrm{str})}(\omega) & =\frac{3 g_{A}^{2}}{32 \pi^{2} F_{\pi}^{2}}\left(M_{\pi_{0}}^{2}-\omega^{2}\right)\left[\omega-2 \sqrt{M_{\pi^{0}}^{2}-\omega^{2}} \arccos \frac{-\omega}{M_{\pi^{0}}}\right] \\
& -\frac{3 g_{A}^{2} \omega}{32 \pi^{2} F_{\pi}^{2}}\left(3 M_{\pi^{0}}^{2}-2 \omega^{2}\right) \ln \frac{M_{\pi^{0}}}{\lambda}+\omega^{3} d_{24}^{r}(\lambda)-8 M_{\pi^{0}}^{2} \omega d_{28}^{r}(\lambda), \\
\Sigma^{(3, \mathrm{em})}(\omega) & =\frac{g_{A}^{2}}{16 \pi^{2} F_{\pi}^{2}}\left[\left(M_{\pi_{+}}^{2}-M_{\pi_{0}}^{2}\right) \omega-2\left\{\left(M_{\pi_{+}}^{2}-\omega^{2}\right)^{3 / 2} \arccos \frac{-\omega}{M_{\pi^{+}}}\right.\right. \\
& \left.-\frac{g_{A}^{2} \omega}{16 \pi^{2} F_{\pi}^{2}}\left[\left(3 M_{\pi^{+}}^{2}-2 \omega^{2}\right) \ln \frac{M_{\pi^{+}}}{M_{\pi^{0}}}+3\left(M^{2}\right)^{3 / 2} \arccos \frac{-M_{\pi_{+}}}{M_{\pi^{0}}}\right\}\right] \\
& -\frac{e^{2}}{16 \pi^{2}}\left(1+\tau_{3}\right)\left[\omega\left(1-\ln \frac{M_{\pi^{0}}}{\lambda}\right]-2 \sqrt{m_{\gamma}^{2}-\omega^{2}} \arccos \frac{-\omega}{m_{\gamma}}\right] \\
& -e^{2} \omega\left[k_{10}^{r}(\lambda)\left(1-\tau^{3}\right)+k_{11}^{r}(\lambda)+k_{12}^{r}(\lambda)\right] .
\end{aligned}
$$

Note for these formulae to be valid, $m_{\gamma}$ must be larger than $\omega$ so that for calculating the mass shift $\Sigma(0)$, one first has to take the limit $\omega \rightarrow 0$ before letting the photon mass vanish. The result for the strong part agrees with the one in [28] (if one identifies $M_{\pi}$ in [28] with $M_{\pi^{0}}$ ). Altogether, a combination of four dimension three (one) em LECs contributes to the isoscalar (isovector) em self-energy. The corresponding third order nucleon mass shift follows as

$$
\begin{aligned}
\delta m^{(3)}=\Sigma^{(3, \mathrm{str})}(0)+\Sigma^{(3, \mathrm{em})}(0) & =-\frac{3 g_{A}^{2} M_{\pi^{0}}^{3}}{32 \pi F_{\pi}^{2}}-\frac{g_{A}^{2}}{16 \pi F_{\pi}^{2}}\left(M_{\pi^{+}}^{3}-M_{\pi^{0}}^{3}\right) \\
& =-13.6-1.0 \mathrm{MeV}
\end{aligned}
$$

To this order, the only em effect comes in via the pion mass difference, i.e. it is given entirely in terms of the LEC $C$. Note that it is the same for the proton and the neutron (as it is expected from our previous arguments) and that it amounts to a $7 \%$ correction to the strong result.

The fourth order self-energy can be worked out along similar lines. The much more tedious calculation is detailed in app. B. Besides the pion loop graphs with unequal masses (i.e. insertions $\left.\sim f_{2}, c_{5}, C\right)$, shown in fig.2, one has to consider photon loop graphs of the tadpole, self-energy and eye graph type, see fig.3. These terms introduce additional photonic counterterms proportional to $m_{\gamma}^{2}$, which have to be constructed according to symmetry arguments and only drop out at the end. The corresponding LECs are denoted by $h_{i, \gamma}$ and the pertinent $\beta$-functions $\delta_{i, \gamma}$. In addition, there are the so-called induced terms from lower orders, which chiefly appear because of the expansion of $\omega$ in powers of $1 / \mathrm{m}$. The renormalization of the self-energy involves combinations of the monomials $\mathcal{O}_{29, \ldots, 34}^{(4)}$ as well as seven equation of motion terms. These and the additional photonic monomials of fourth order are

$$
\mathcal{O}_{91}^{(4)}=\overleftarrow{\nabla}_{\mu}\left\langle Q_{+}^{2}-Q_{-}^{2}\right\rangle \nabla^{\mu}, \quad \mathcal{O}_{92}^{(4)}=\overleftarrow{\nabla}_{\mu}\left\langle Q_{+}^{2}+Q_{-}^{2}\right\rangle \nabla^{\mu}, \quad \mathcal{O}_{93}^{(4)}=v \cdot \overleftarrow{\nabla}\left\langle Q_{+}^{2}-Q_{-}^{2}\right\rangle v \cdot \nabla
$$




$$
\begin{aligned}
& \mathcal{O}_{94}^{(4)}=v \cdot \overleftarrow{\nabla}\left\langle Q_{+}^{2}+Q_{-}^{2}\right\rangle v \cdot \nabla, \quad \mathcal{O}_{95}^{(4)}=\overleftarrow{\nabla}_{\mu}\left\langle Q_{+}\right\rangle \tilde{Q}_{+} \nabla^{\mu}, \quad \mathcal{O}_{96}^{(4)}=v \cdot \overleftarrow{\nabla}\left\langle Q_{+}\right\rangle \tilde{Q}_{+} v \cdot \nabla \\
& \mathcal{O}_{1, \gamma}^{(4)}=m_{\gamma}^{2}\left\langle Q_{+}\right\rangle \tilde{Q}_{+}, \quad \mathcal{O}_{2, \gamma}^{(4)}=m_{\gamma}^{2}\left\langle Q_{+}^{2}+Q_{-}^{2}\right\rangle, \quad \mathcal{O}_{3, \gamma}^{(4)}=m_{\gamma}^{2}\left\langle Q_{+}^{2}-Q_{-}^{2}\right\rangle
\end{aligned}
$$

and the pertinent $\beta$-functions $\delta_{i}$ are given by

$$
\begin{aligned}
\delta_{1+2+3} & =32 f_{1} Z-12\left(f_{1}+f_{3}\right) g_{A}^{2} Z-4\left(f_{1}+f_{2}+f_{3}\right)+\frac{4}{m} g_{A}^{2} Z^{2}+\frac{6}{m} g_{A}^{2} Z^{2}-16 Z^{2}\left(c_{2}-\frac{g_{A}^{2}}{8 m}+4 c_{3}\right), \\
\delta_{4+5} & =12 g_{A}^{2} Z-4\left(f_{1}+f_{2}+f_{3}\right)+8 f_{2} Z \quad \delta_{29+30}=12 g_{A}^{2} Z-4 c_{5}, \quad \delta_{31}=-2 c_{5}, \\
\delta_{32+33} & =-8 Z c_{3}+4(2 Z-1) c_{1}-2 Z\left(c_{2}-\frac{g_{A}^{2}}{8 m}\right)+\frac{5 g_{A}^{2}}{4 m} Z+2 c_{1} g_{A}^{2} Z-\frac{9}{8}\left(f_{1}+f_{3}\right)+2 f_{1}, \\
\delta_{34} & =-4 c_{1}+\frac{1}{2} f_{2}\left(1+\frac{3}{4} g_{A}^{2}\right), \quad \delta_{91+92}=-\frac{2}{m}+\frac{g_{A}^{2}}{m} Z, \quad \delta_{93+94}=\frac{4}{m}-3 f_{2}, \\
\delta_{95} & =-\frac{2}{m}, \quad \delta_{96}=\frac{4}{m}+9\left(f_{1}+f_{3}\right)-\frac{4 g_{A}^{2}}{m} Z, \quad \delta_{1, \gamma}=-\frac{2}{m}, \quad \delta_{2+3, \gamma}=-\frac{2}{m} .
\end{aligned}
$$

The size of the finite pieces of these new counterterms follows from the considerations of section 2.4. $h_{j}^{r}\left(M_{\rho}\right)=\mathcal{O}(1) /(4 \pi)(j=91, \ldots, 96)$. In addition to these, one needs the term 22 of table 1 in ref. 25] to account for the isospin violation through the light quark mass difference. The corresponding electromagnetic self-energy is given in app. B. Here, we concentrate on the em mass shift to fourth order, which has the general structure

$$
\begin{aligned}
\delta m^{(4, \mathrm{em})} & =\Sigma^{(4, \mathrm{em})}(0)+\Sigma^{(4, \text { induced })} \\
\Sigma^{(4, \text { induced })} & =-\frac{l^{2}}{2 m} \Sigma^{(3, \mathrm{em})^{\prime}}(0)+\delta m^{(2)} \cdot \Sigma^{(3, \mathrm{em})^{\prime}}(0)+\delta m^{(2, \mathrm{em})} \cdot \Sigma^{(3, \mathrm{str})^{\prime}}(0),
\end{aligned}
$$

using the well-known result for the mass shift to second order,

$$
\delta m^{(2)}=-4 c_{1} M_{\pi^{0}}^{2}+\delta m^{(2, \mathrm{em})}=-4 c_{1} M_{\pi^{0}}^{2}+\left\{-2 B\left(m_{u}-m_{d}\right) c_{5} \tau^{3}+\frac{1}{2} e^{2}\left(f_{1}+f_{3}\right)-\frac{1}{2} e^{2} \tau^{3} f_{2}\right\} .
$$

It is important to note that the induced mass shift cancels exactly the strong and photonic eye graph contribution with insertions $\sim c_{1,5}, f_{1,2,3}$ (for details, see app. B). As already noted in ref. 28, , the so calculated mass shift contains momentum dependent parts and terms $\sim \ln \left(M_{\pi^{0}} / \lambda\right)$. With a proper redefinition of the LECs,

$$
h_{i,(\gamma)}^{r}(\lambda)=\frac{\delta_{i,(\gamma)}}{16 \pi^{2}}\left[\bar{h}_{i,(\gamma)}+\ln \frac{M_{\pi^{0}}}{\lambda}\right],
$$

one can eliminate all these logarithms and also the $l^{2}$-dependent terms vanish. This finally gives

$$
\begin{aligned}
\delta m^{(4)} & =\delta m^{(4, \mathrm{str})}+\delta m^{(4, \mathrm{em})} \\
\delta m^{(4, \mathrm{em})} & =\frac{1}{32 \pi^{2} F_{\pi}^{2}}\left(\frac{c_{2}}{2}-2 \frac{g_{A}^{2}}{m}\right)\left(M_{\pi^{+}}^{4}-M_{\pi^{0}}^{4}\right) \\
& +\frac{M_{\pi^{+}}^{2}}{16 \pi^{2} F_{\pi}^{2}}\left(8 c_{1} M_{\pi^{0}}^{2}-\frac{g_{A}^{2}}{m} M_{\pi^{+}}-\left(c_{2}+4 c_{3}\right) M_{\pi^{+}}^{2}+4 e^{2} f_{1}+e^{2} f_{2} \tau^{3}\right) \ln \frac{M_{\pi^{+}}}{M_{\pi^{0}}}
\end{aligned}
$$

${ }^{\# 7}$ Here, $\delta_{n+m}$ stands for $\delta_{n}+\delta_{m}$.

${ }^{\# 8}$ We have also calculated the strong fourth order mass shift and find agreement with the result of ref. [28] if one identifies $M_{\pi}$ with the neutral pion mass as argued before. 
with $\delta m^{(4, \mathrm{str})}$ given in eq.(3.22) of ref. 228]. Setting $f_{1}= \pm 1 /(4 \pi)$, the numerical value of the em fourth order mass shift is tiny, for the proton we find $\delta m_{p}^{(4, \mathrm{em})}=-0.10 \ldots-0.01 \mathrm{MeV}$ and for the neutron $\delta m_{n}^{(4, \mathrm{em})}=-0.06 \ldots+0.03 \mathrm{MeV}$. This is completely negligible and comparable to the fourth order mass shift, which was shown to be less than $0.1 \mathrm{MeV}$ in ref.[28].

\subsection{Scalar form factor}

The scalar form factor of the nucleon is defined via

$$
\left\langle N\left(p^{\prime}\right)\left|m_{u} \bar{u} u+m_{d} \bar{d} d\right| N(p)\right\rangle=\bar{u}\left(p^{\prime}\right) u(p) \sigma(t), \quad t=\left(p^{\prime}-p\right)^{2},
$$

for a nucleon state $|N(p)\rangle$ of four-momentum $p$. At $t=0$, which gives the much discussed pionnucleon $\sigma$-term, one can relate this matrix element to the so-called strangeness content of the nucleon. A direct determination of the $\sigma$-term is not possible, but rather one extends pion-nucleon scattering data into the unphysical region and determines $\sigma_{\pi N}\left(t=2 M_{\pi}^{2}\right)$, i.e. at the so-called Cheng-Dashen point. The relation to the $\sigma$-term is given by the low-energy theorem of Brown, Peccei and Pardee [30],

$$
\sigma_{\pi N}\left(2 M_{\pi}^{2}\right)=\sigma_{\pi N}(0)+\Delta \sigma_{\pi N}+\Delta R
$$

where $\Delta \sigma_{\pi N}$ parametrizes the $t$-dependence of the sigma-term whereas $\Delta R$ is a remainder not fixed by chiral symmetry. The most systematic determination of $\Delta \sigma_{\pi N}$ has been given in ref. [31] (although there has been much debate recently about the pion-nucleon data basis and alike), $\Delta \sigma_{\pi N}=(15 \pm 1) \mathrm{MeV}$. The remainder $\Delta R$ has been bounded in ref. [32], $\Delta R<2 \mathrm{MeV}$. In the case of isospin violation, one has of course to differentiate between the proton and the neutron $\sigma$-terms, as detailed in ref.[B]. There, it was shown that the third order effects can shift the proton $\sigma$-term by about $8 \%$ and have a smaller influence on the shift to the Cheng-Dashen (CD) point. Here, we work out explicitly the isospin violating corrections to this shift to fourth order. This is motivated by the fact that in the difference most of the counterterm contributions drop out, more precisely, only momentum-dependent contact terms can contribute to the shift. Such terms only appear at fourth order since due to parity one needs two derivatives and any quark mass or em insertion accounts for at least two orders. Before proceeding, we have to discuss a subtlety related to the definition of the CD-point. Generally, it is taken to be at $t=2 M_{\pi^{+}}^{2}$. For the reasons given before, we choose to work with $t=2 M_{\pi^{0}}^{2}$ to define this point in the unphysical plane. In what follows, this should be kept in mind (note also that the small effect between these two values of squared momentum transfer can readily be evaluated).

We now evaluate the scalar form factor for the proton $\left(\tau_{3}=1\right)$. It can be decomposed as

$$
\sigma_{\pi N}^{(4)}(t)=\sigma_{\pi N}^{(4), \mathrm{IC}}(t)+\sigma_{\pi N}^{(4), \mathrm{IV}}(t) .
$$

The isospin-conserving strong terms have already been evaluated in ref.[32]. Here, we concentrate on the em corrections $\sim 1$ (in isospin space) and all terms $\sim \tau_{3}$. Consider first the IV terms. The pertinent one loop graphs shown in fig. We have eye graphs and tadpoles with insertions $\sim f_{2}, c_{5}$. These can be evaluated straightforwardly and give the terms which explicitly depend on 
$\tau_{3}$,

$$
\begin{aligned}
\sigma_{\pi N}^{\text {eye,IV }}(t) & =\left(2 B\left(m_{u}-m_{d}\right) c_{5}-\frac{1}{2} e^{2} f_{2} F_{\pi}^{2}\right) \frac{g_{A}^{2}}{4 F_{\pi}^{2}} \\
& \times\left[2 M_{\pi^{+}}^{2}\left(\Omega_{3}^{+}(t)+t\left(\Omega_{4}^{+}(t)+\Omega_{2}^{+}(t)\right)\right)-M_{\pi^{0}}^{2}\left(\Omega_{3}^{+}(t)+t\left(\Omega_{4}^{0}(t)+\Omega_{2}^{+}(t)\right)\right)\right] \tau_{3} \\
\sigma_{\pi N}^{\mathrm{tad}, \mathrm{IV}}(t) & =-\left[B\left(m_{u}-m_{d}\right) c_{5} I_{0}^{0}(t) \frac{M_{\pi^{0}}^{2}}{F_{\pi}^{2}}+\frac{1}{2} e^{2} f_{2} I_{0}^{+}(t) M_{\pi^{+}}^{2}\right] \tau_{3}
\end{aligned}
$$

in terms of standard loop functions with the appropriate pion masses in the propagators as indicated by the superscripts. The momentum dependence of these equations can be expressed entirely in terms of

$$
\begin{aligned}
I_{0}\left(2 M_{\pi}^{2}\right)-I_{0}(0) & =-\frac{1}{8 \pi^{2}}\left\{\frac{\pi}{4}-1\right\}=0.0027, \\
-\left.\frac{t}{4} \Omega_{2}(t)\right|_{t=2 M_{\pi}^{2}} & =\frac{\sqrt{2} M_{\pi}}{128 \pi} \ln \frac{2-\sqrt{2}}{2+\sqrt{2}}=-0.0062 M_{\pi},
\end{aligned}
$$

evaluated for the appropriate pion mass appearing in the loop functions. There is no corresponding tadpole with a photon loop since there is no vertex with two photons and a scalar source. Furthermore, the photonic eye graph with a $c_{5}$-insertion only gives a constant contribution, i.e. it drops out in the scalar form factor. As counterterm contributions we have terms of order $p^{4}$, of order $e^{2} p^{2}$ and form $\mathcal{O}\left(e^{4}\right)$ which contribute to the $\sigma$-term. But here we only need the $t$-dependent terms and so we are left with one new LEC,

$$
\Delta \sigma_{\pi N}^{\mathrm{ct}, \mathrm{IV}}=e^{2} M_{\pi}^{2} h_{95}^{r}(\lambda) \tau_{3}
$$

There is also a strong fourth order counterterm $\sim \overleftarrow{\nabla}_{\mu} \tilde{\chi}_{+} \nabla^{\mu}$, which has a finite low energy constant $d$ and therefore does not appear in table 1 of ref. 225]. Its effect, however, can be completely absorbed in a redefinition of the dimension two LEC $c_{5}$,

$$
c_{5} \rightarrow c_{5}+\frac{4 M_{\pi}^{2} d}{\left(4 \pi F_{\pi}\right)^{2}}
$$

and is thus unobservable. In what follows, we always imply that any contribution from the strong term $\sim d$ is absorbed in $c_{5}$ (a similar remark holds for the term $\sim d_{22}$, see the next section). Consider now the corresponding em terms $\sim f_{1,3}$ (i.e. the same loop graphs as in fig. 团) which give a contribution $\sim 1$ and the pertinent counterterms. These give

$$
\begin{aligned}
\sigma_{\pi N}^{(4), \mathrm{IC}}(t)= & \frac{1}{2} e^{2}\left(f_{1}+f_{3}\right) \frac{g_{A}^{2}}{4}\left[2 M_{\pi^{+}}^{2}\left(\Omega_{3}^{+}(t)+t\left(\Omega_{4}^{+}(t)+\Omega_{2}^{+}(t)\right)\right)\right. \\
& \left.+M_{\pi^{0}}^{2}\left(\Omega_{3}^{+}(t)+t\left(\Omega_{4}^{0}(t)+\Omega_{2}^{+}(t)\right)\right)\right]-2 e^{2} f_{1} I_{0}^{+}(t) M_{\pi^{+}}^{2} \\
& +\frac{1}{4} e^{4}\left(h_{1}^{r}+h_{2}^{r}+h_{3}^{r}\right) F_{\pi}^{2}+2 e^{2} M_{\pi^{0}}^{2}\left(h_{32}^{r}+h_{33}^{r}\right)+\frac{1}{2} e^{2} t\left(h_{91}^{r}+h_{93}^{r}\right) .
\end{aligned}
$$

We now turn towards the numerical analysis of these formulae. Consider first the IV terms. Because of the tiny coefficients appearing in the evaluation of the loop contributions, cf. eq.(3.15), 
these are only fractions of an $\mathrm{MeV}, \Delta \sigma_{\pi N}^{4, I V \text {,loop }}=-0.05 \mathrm{MeV}$ and can thus be completely neglected. For the counterterm contributions, setting all appearing LECs on the values obtained from dimensional analysis as explained in section 2.4, one finds a total contribution $\Delta \sigma_{\pi N}^{4, I V, \text { ct }}= \pm 0.01 \mathrm{MeV}$. For the IC em terms, we find (setting again $f_{1,3}= \pm 1 / 4 \pi$ ) a completely negligible loop contribution (less than $0.01 \mathrm{MeV}$ ) and the counterterms give $\pm 0.7 \mathrm{MeV}$ for the LECs estimated from dimensional analysis. Note, however, that if the numerical factors $\tilde{f}_{1,3}$ are somewhat bigger than one, one could easily have a shift of $\pm 2 \mathrm{MeV}$, which is a substantial electromagnetic effect.

\subsection{Neutral pion scattering off nucleons}

As pointed out long time ago by Weinberg [7], the difference in the S-wave scattering lengths for neutral pions off nucleons is sensitive to the light quark mass difference,

$$
\begin{aligned}
a\left(\pi^{0} p\right)-a\left(\pi^{0} n\right) & =\frac{1}{4 \pi} \frac{1}{1+M_{\pi^{+}} / m_{p}} \frac{-4 B\left(m_{u}-m_{d}\right) c_{5}}{F_{\pi}^{2}}+\mathcal{O}\left(q^{3}\right) \\
& =\frac{1}{4 \pi} \frac{1}{1+M_{\pi^{+}} / m_{p}} \Delta_{2}\left(M_{\pi^{0}}\right)+\mathcal{O}\left(q^{3}\right) .
\end{aligned}
$$

It was shown in ref. [8] by an explicit calculation that to third order there are no corrections to this formula. This is based on the fact that the electromagnetic Lagrangian can not contribute at this order since the charge matrix has to appear quadratic and never two additional pions can appear. However, at next order one can of course have loop graphs with one dimension two insertion and additional em counterterms. To obtain the first correction to Weinberg's prediction, eq.(3.20), one thus has to compute the fourth order corrections as it is done here. ${ }^{* 9}$ These are due to strong dimension two insertions $\sim c_{5}$ and em insertions $\sim f_{2}$ as shown in fig.5. For the difference $a\left(\pi^{0} p\right)-a\left(\pi^{0} n\right)$ we only have to consider the operators $\sim \tau^{3}$. While all six type of graphs contribute to the strong isospin violation, the graphs 2 ) and 4) do not have a contribution $\sim f_{2}$ because this operator does not couple to neutral pions. Expressed in terms of standard loop functions, we find (the counterterm contribution is discussed below)

$$
\begin{aligned}
\Delta_{4}(\omega) & =\Delta_{2}(\omega)\left(1+\Delta_{2}^{\mathrm{str}}(\omega)+R \Delta_{2}^{\mathrm{em}}(\omega)\right)+\mathcal{O}\left(q^{5}\right) \\
\Delta_{2}^{\mathrm{str}}(\omega) & =\frac{1}{2 F_{\pi}^{2}}\left[\left(J_{1}^{+}(\omega)+J_{1}^{+}(-\omega)\right)+\omega\left(J_{0}^{+}(\omega)-J_{0}^{+}(-\omega)\right)\right] \\
& +\frac{1}{2 F_{\pi}^{2}}\left[M_{\pi^{0}}^{2} I_{0}^{0}-8 I_{2}^{0}+\Delta_{\pi}^{+}+3 \Delta_{\pi}^{0}\right] \\
& -\frac{1}{8 F_{\pi}^{2}}\left[\omega^{2}\left(G_{0}^{+}(\omega)+G_{0}^{+}(-\omega)\right)+2 \omega\left(G_{2}^{+}(\omega)+G_{2}^{+}(-\omega)\right)+\left(G_{3}^{+}(\omega)+G_{3}^{+}(-\omega)\right)\right] \\
& +\frac{3 g_{A}^{2}}{4 F_{\pi}^{2}}\left[3 \tilde{\Gamma}_{2}^{0}(0)-2 \tilde{\Gamma}_{2}^{+}(0)-2 M_{\pi^{+}}^{2} \Omega_{3}^{+}(0)-M_{\pi^{0}}^{2} \Omega_{3}^{0}(0)+2 G_{2}^{0}(0)\right] \\
\Delta_{2}^{\mathrm{em}}(\omega) & =\frac{1}{2 F_{\pi}^{2}}\left[\left(J_{1}^{+}(\omega)+J_{1}^{+}(-\omega)\right)+\omega\left(J_{0}^{+}(\omega)-J_{0}^{+}(-\omega)\right)+2 \Delta_{\pi}^{+}\right]
\end{aligned}
$$

\footnotetext{
${ }^{\# 9}$ As in refs. [7, 8], we neglect photon loops and soft photon radiation off the protons. These would affect the Weinberg prediction and our result in a similar fashion.
} 


$$
\begin{aligned}
& +\frac{1}{8 F_{\pi}^{2}}\left[\omega^{2}\left(G_{0}^{+}(\omega)+G_{0}^{+}(-\omega)\right)+2 \omega\left(G_{2}^{+}(\omega)+G_{2}^{+}(-\omega)\right)+\left(G_{3}^{+}(\omega)+G_{3}^{+}(-\omega)\right)\right] \\
& -\frac{3 g_{A}^{2}}{4 F_{\pi}^{2}}\left[2 \tilde{\Gamma}_{2}^{0}(0)+2 M_{\pi^{+}}^{2} \Omega_{3}^{+}(0)+M_{\pi^{0}}^{2} \Omega_{3}^{0}(0)-2 G_{2}^{0}(0)\right] \\
R & =e^{2} f_{2} / \Delta_{2} .
\end{aligned}
$$

where the superscripts ',$+ 0^{\prime}$ on the loop functions refer to the pion mass with which these have to be evaluated (for the precise definitions, see the review [21]). At threshold $\omega=M_{\pi^{0}}$, these functions can readily be evaluated. Adding also the pertinent counterterms, one gets

$$
\begin{aligned}
\Delta_{2}^{\mathrm{str}}= & \frac{1}{16 \pi^{2} F_{\pi}^{2}}\left\{-M_{\pi^{0}}^{2} \ln \frac{M_{\pi^{0}}}{\lambda}+\frac{1}{2} M_{\pi^{+}}^{2} \ln \frac{M_{\pi^{+}}}{M_{\pi^{0}}}+\frac{3}{2} M_{\pi^{+}}^{2} \ln \frac{M_{\pi^{0}}}{\lambda}\right. \\
& \left.-\frac{M_{\pi^{0}} M_{\pi^{+}}^{2}}{\sqrt{M_{\pi^{+}}^{2}-M_{\pi^{0}}^{2}}}\left[\arccos \frac{-M_{\pi^{0}}}{M_{\pi^{+}}}-\arccos \frac{M_{\pi^{0}}}{M_{\pi^{+}}}\right]\right\} \\
+ & \frac{3 g_{A}^{2}}{16 \pi^{2} F_{\pi}^{2}}\left\{-\frac{1}{4} M_{\pi^{+}}^{2}-3 M_{\pi^{0}}^{2} \ln \frac{M_{\pi^{0}}}{\lambda}-\frac{1}{8} M_{\pi^{0}}^{2}\right\} \\
\Delta_{2}^{\mathrm{em}}= & \frac{1}{16 \pi^{2} F_{\pi}^{2}}\left\{M_{\pi^{0}}^{2}\left(4-15 \ln \frac{M_{\pi^{0}}}{\lambda}-16 \ln \frac{M_{\pi^{+}}}{M_{\pi^{0}}}\right)+M_{\pi^{+}}^{2}\left(\frac{5}{2} \ln \frac{M_{\pi^{0}}}{\lambda}+\frac{7}{2} \ln \frac{M_{\pi^{+}}}{M_{\pi^{0}}}\right)\right. \\
& \left.-\frac{M_{\pi^{0}}\left(6 M_{\pi^{+}}^{2}-8 M_{\pi^{0}}^{2}\right)}{\sqrt{M_{\pi^{+}}^{2}-M_{\pi^{0}}^{2}}}\left[\arccos \frac{-M_{\pi^{0}}}{M_{\pi^{+}}}-\arccos \frac{M_{\pi^{0}}}{M_{\pi^{+}}}\right]\right\} \\
+ & \frac{3 g_{A}^{2}}{16 \pi^{2} F_{\pi}^{2}}\left\{M_{\pi^{0}}^{2}\left(\frac{1}{4}+2 \ln \frac{M_{\pi^{0}}}{\lambda}\right)+2 M_{\pi^{+}}^{2}\left(\frac{1}{4}+\ln \frac{M_{\pi^{0}}}{\lambda}+\ln \frac{M_{\pi^{+}}}{M_{\pi^{0}}}\right)\right\} \\
\Delta_{4}^{\mathrm{ct}}\left(M_{\pi^{0}}\right)= & -\frac{2}{F_{\pi}^{2}} B\left(m_{u}-m_{d}\right)\left\{e^{2}\left(h_{29}^{r}(\lambda)+h_{30}^{r}(\lambda)\right)\right\}
\end{aligned}
$$

where we have no exhibited the strong fourth order contribution proportional to $d_{22}$ since it can be absorbed in the $c_{5}$ term and is thus unobservable as discussed before. Consider first the loop contributions. Since we can not fix the counterterms from data, we are left with a spurious scale dependence which reflects the theoretical uncertainty at this order. For $\lambda=\{0.5,0.77,1.0\} \mathrm{GeV}$ we find

$$
\Delta_{2}^{\text {str }}=\{-7.1,0.9,5.7\} \cdot 10^{-2}, \quad \Delta_{2}^{\mathrm{em}}=\{11.5,12.0,12.3\} \cdot 10^{-2} .
$$

In both cases, the dominant contribution comes from the rescattering graphs 5) which is largely canceled the pion rescattering diagrams 6 ). The counterterms are estimated based on dimensional analysis at the scale $\lambda=M_{\rho}$ and give a contribution of about $-0.3 \cdot 10^{-2}$. Even if the LECs $h_{29}^{r}(\lambda)+h_{30}^{r}(\lambda)$ would be a factor of ten larger than assumed, the counterterm contribution would not exceed $\pm 3 \%$. Altogether, the correction Weinberg's prediction, eq.(3.20), are in the range of 4 to 18 percent, i.e. fairly small. We do not attempt here to calculate the isoscalar fourth order corrections since at present the sizeable uncertainty in the determination of the isoscalar scattering lengths extracted from level shifts in pionic hydrogen and deuterium is larger than this isospinviolating effect (for a detailed discussion, see e.g. ref. [9]). Finally, we wish to mention that in ref. 33] isospin-violation for neutral pion photoproduction off nucleons was discussed which allows 
one to eventually measure directly the very small $\pi^{0} p$ scattering length by use of the final-state theorem.

\section{Summary and conclusions}

In this paper, we have considered baryon chiral perturbation theory in the presence of virtual photons to fourth order in small momenta $q$ (counting the electric charge as a small parameter $e \sim q)$. The pertinent results of this investigation can be summarized as follows:

i) We have constructed the complete fourth order electromagnetic Lagrangian including up to four nucleon (quark) charge matrices. We have omitted all terms which only lead to an overall mass shift or coupling constant renormalization. The corresponding terms are collected in section 2.3 and in app. Q.

ii) To get an estimate of the novel electromagnetic low-energy constants, we have performed dimensional analysis and argued that measured in appropriate powers of the inverse scale of chiral symmetry breaking, these should be of order $1 / 4 \pi$ or $1 /(4 \pi)^{2}$.

iii) We have evaluated the third order isospin-violating nucleon mass shift and found that amounts to a seven percent correction of its strong (isospin-conserving) counterpart. The fourth order electromagnetic mass shift is tiny (as is the strong fourth order mass shift).

iv) The electromagnetic isospin-conserving contributions to the scalar form factor of the proton (and neutron) are of the order $1 \ldots 2 \mathrm{MeV}$, i.e. not completely negligible and are thus of (minor) relevance in connecting the $\sigma$-term at the Cheng-Dashen point to its value at zero momentum transfer. On the other hand, all isospin-violating contributions are very small, only fractions of an $\mathrm{MeV}$.

v) We have worked out the first corrections to Weinbergs time-honored prediction for the difference of the S-wave scattering for the neutral pions off nucleons. These corrections are small, we estimate their numerical value in the range from 4 to $18 \%$.

\section{Acknowledgments}

We are grateful to Evgeni Epelbaum for some useful comments.

\section{A Electromagnetic meson Lagrangian}

Here we briefly review the effective electromagnetic meson Lagrangian, following ref. [5]. The effective field theory build of pions, collected in $U(x)=u^{2}(x)$, photons $\left(A_{\mu}\right)$ and other scalar $(s)$, pseudoscalar $(p)$, vector $\left(v_{\mu}\right)$ and axial-vector $\left(a_{\mu}\right)$ external sources starts at dimension two,

$$
\mathcal{L}_{\pi \pi}^{(2)}=-\frac{1}{4} F_{\mu \nu} F^{\mu \nu}-\frac{\lambda}{2}\left(\partial_{\mu} A^{\mu}\right)^{2}+\frac{F^{2}}{4}\left\langle\mathrm{~d}_{\mu} U \mathrm{~d}^{\mu} U^{\dagger}+\chi U^{\dagger}+\chi^{\dagger} U\right\rangle+C\left\langle Q_{R} U Q_{L} U^{\dagger}\right\rangle
$$


with $F=88 \mathrm{MeV}$ the pion decay constant in the chiral limit, $F_{\mu \nu}=\partial_{\mu} A_{\nu}-\partial_{\nu} A_{\mu}$ denotes the photon field strength tensor, $\lambda$ is the gauge-fixing parameter (from here on, we work in the Landau gauge $\lambda=1$ ), $\mathrm{d}_{\mu}$ the generalized covariant derivative (given in eq.(2.27)) and $Q$ is the quark charge matrix, see eq.(2.14). It is advantageous to work in the so-called $\sigma$-model gauge,

$$
U(x)=\sigma(x) \mathbf{1}+i \vec{\tau} \cdot \vec{\pi}(x) / F, \quad \sigma(x)=\sqrt{1-\pi^{2}(x) / F^{2}} .
$$

The third term in eq. A.1) is the standard non-linear $\sigma$-model coupled to external sources (we neglect here singlet components and set $\left\langle a_{\mu}\right\rangle=\left\langle v_{\mu}\right\rangle=0$ ). The last term in the dimension two Lagrangian is the lowest order chiral invariant term one can construct from pion and photon fields [1]. To make it invariant under chiral $\mathrm{SU}(2)_{L} \times \mathrm{SU}(2)_{R}$ transformations, one has introduced the spurions $Q_{L, R}$ given in eq.(2.15). The constant $C$ can be calculated from the neutral to charged pion mass difference since this term leads to

$$
\left(M_{\pi^{+}}^{2}-M_{\pi^{0}}^{2}\right)_{\mathrm{em}} \equiv\left(\delta M_{\pi^{ \pm}}^{2}\right)_{\mathrm{em}}=\frac{2 e^{2} C}{F^{2}}, \quad C=5.9 \cdot 10^{-5} \mathrm{GeV}^{4} .
$$

This identification is based upon the fact that the quark mass difference $m_{d}-m_{u}$ only gives a tiny contribution to $M_{\pi^{+}}^{2}-M_{\pi^{0}}^{2}$ due $\pi^{0}-\eta$-mixing. Note that in the $\sigma$-model gauge, this is the only contribution from the term $\sim C$. It is useful to introduce the dimensionless constant $Z=C / F^{4}=0.89$. As already stated before, we count the external vector and axial-vector fields as well as the charge matrices $Q, Q_{L}, Q_{R}$ as $\mathcal{O}(q)$ and the photon field as $\mathcal{O}(1)$. This has the advantage of a consistent power counting between the strong and electromagnetic interactions, i.e. $e \sim q$ and one has terms of dimension two, four and so on. Here, dimension two means either order $q^{2}$ or $e^{2}$ and similar at higher orders. The fourth order em Lagrangian for SU(2) has been developed in ref. [5]. It is a sum of 13 local operators quadratic in the charge matrix,

$$
\mathcal{L}_{\pi \pi, \mathrm{em}}^{(4)}=\sum_{i=1}^{13} k_{i} \mathcal{O}_{i}
$$

with the $\mathcal{O}_{i}$ monomials in the fields of dimension four. The low-energy constants $k_{i}$ absorb the divergences in the standard manner,

$$
k_{i}=\kappa_{i} L+k_{i}^{r}(\mu)
$$

with $L$ given in eq.(2.32). The corresponding expressions for the $O_{i}$ and the corresponding $\beta-$ functions $\kappa_{i}$ are given in [5]. More details can be found in ref. [6].

\section{B Self-energy of the nucleon to fourth order}

In this appendix, we give the explicit result for the em contribution to the nucleons' self-energy to fourth order, which follows after straightforward but tedious calculation of the diagrams shown in figs.1.2, the induced terms from the lower orders and the pertinent counterterms. The resulting lengthy expression takes the form (using as always dimensional regularization)

$$
\Sigma^{(4, e m)}(\omega)=\sum_{i=1}^{7} \Gamma_{i} \tilde{\Sigma}_{i}^{(4, e m)}(\omega)
$$


with

$$
\begin{aligned}
& \tilde{\Sigma}_{1}^{(4, e m)}(\omega)=\left\{\quad\left(l^{2}-2 \omega^{2}+8 m c_{1} M_{\pi^{0}}^{2}\right)\left[3 \omega \sqrt{M_{\pi^{+}}^{2}-\omega^{2}} \arccos \frac{-\omega}{M_{\pi^{+}}}-3 \omega \sqrt{M_{\pi^{0}}^{2}-\omega^{2}} \arccos \frac{-\omega}{M_{\pi^{0}}}\right]\right. \\
& \text { - } \frac{1}{2}\left(M_{\pi^{+}}^{2}-M_{\pi^{0}}^{2}\right)\left[l^{2}-\omega^{2}+8 m c_{1} M_{\pi^{0}}^{2}+M_{\pi^{+}}^{2}+M_{\pi^{0}}^{2}\right] \\
& +3 M_{\pi^{+}}^{2} \omega \sqrt{M_{\pi^{+}}^{2}-\omega^{2}} \arccos \frac{-\omega}{M_{\pi^{+}}}-3 M_{\pi^{0}}^{2} \omega \sqrt{M_{\pi^{0}}^{2}-\omega^{2}} \arccos \frac{-\omega}{M_{\pi^{0}}} \\
& -\frac{3}{2}\left(M_{\pi^{+}}^{2}-M_{\pi^{0}}^{2}\right)\left[l^{2}-4 \omega^{2}+8 m c_{1} M_{\pi^{0}}^{2}+M_{\pi^{+}}^{2}+M_{\pi^{0}}^{2}\right] \ln \frac{M_{\pi^{0}}}{\lambda} \\
& +\frac{3}{8}\left(M_{\pi^{+}}^{4}-M_{\pi^{0}}^{4}\right) \ln \frac{M_{\pi^{0}}}{\lambda}+\frac{3}{8} M_{\pi^{+}}^{4} \ln \frac{M_{\pi^{+}}}{M_{\pi^{0}}} \\
& \left.+\frac{1}{2}\left[l^{2}-2 \omega^{2}+8 m c_{1} M_{\pi^{0}}^{2}+M_{\pi^{+}}^{2}\right]\left(6 \omega^{2}-3 M_{\pi^{+}}^{2}\right) \ln \frac{M_{\pi^{+}}}{M_{\pi^{0}}}\right\}, \\
& \tilde{\Sigma}_{2}^{(4, e m)}(\omega)=\left\{\left[8 c_{1} M_{\pi^{0}}^{2}-\left(c_{2}-\frac{g_{A}^{2}}{8 m}\right) M_{\pi^{+}}^{2}-4 c_{3} M_{\pi^{+}}^{2}\right] M_{\pi^{+}}^{2} \ln \frac{M_{\pi^{+}}}{M_{\pi^{0}}}\right. \\
& +\left[8 c_{1} M_{\pi^{0}}^{2}-\left(c_{2}+4 c_{3}-\frac{g_{A}^{2}}{8 m}\right)\left(M_{\pi^{+}}^{2}+M_{\pi^{0}}^{2}\right)\right]\left(M_{\pi^{+}}^{2}-M_{\pi^{0}}^{2}\right) \ln \frac{M_{\pi^{0}}}{\lambda} \\
& +\frac{1}{64 \pi^{2}}\left(M_{\pi^{+}}^{4}-M_{\pi^{0}}^{4}\right) c_{2}, \\
& \tilde{\Sigma}_{3}^{(4, e m)}(\omega)=\left\{\frac{1}{2} e^{2}\left(f_{1}+f_{3}\right)\right\} \times \\
& \left\{\frac{1}{2}\left(-6 M_{\pi^{+}}^{2}-3 M_{\pi^{0}}^{2}+18 \omega^{2}\right) \ln \frac{M_{\pi^{0}}}{\lambda}+\frac{1}{2}\left(-6 M_{\pi^{+}}^{2}+12 \omega^{2}\right) \ln \frac{M_{\pi^{+}}}{M_{\pi^{0}}}\right. \\
& +3 \omega\left[2 \sqrt{M_{\pi^{+}}^{2}-\omega^{2}} \arccos \frac{-\omega}{M_{\pi^{+}}}+\sqrt{M_{\pi^{0}}^{2}-\omega^{2}} \arccos \frac{-\omega}{M_{\pi^{0}}}\right] \\
& \left.-\frac{1}{2}\left(2 M_{\pi^{+}}^{2}+M_{\pi^{0}}^{2}+3 \omega^{2}\right)\right\}, \\
& \tilde{\Sigma}_{4}^{(4, e m)}(\omega)=\left\{2 B\left(m_{u}-m_{d}\right) c_{5}+\frac{1}{2} e^{2} f_{2}\right\} \times \\
& \left\{\frac{1}{2}\left(-3 M_{\pi^{0}}^{2}+6 M_{\pi^{+}}^{2}-6 \omega^{2}\right) \ln \frac{M_{\pi^{0}}}{\lambda}+\frac{1}{2}\left(6 M_{\pi^{+}}^{2}-12 \omega^{2}\right) \ln \frac{M_{\pi^{+}}}{M_{\pi^{0}}}\right. \\
& +3 \omega\left[-2 \sqrt{M_{\pi^{+}}^{2}-\omega^{2}} \arccos \frac{-\omega}{M_{\pi^{+}}}+\sqrt{M_{\pi^{0}}^{2}-\omega^{2}} \arccos \frac{-\omega}{M_{\pi^{0}}}\right] \\
& \left.-\frac{1}{2}\left(-2 M_{\pi^{+}}^{2}+M_{\pi^{0}}^{2}-\omega^{2}\right)\right\}, \\
& \tilde{\Sigma}_{5}^{(4, e m)}(\omega)=\left[4 e^{2} f_{1} M_{\pi^{+}}^{2}+\left(2 B\left(m_{u}-m_{d}\right) c_{5}+e^{2} f_{2} M_{\pi^{+}}^{2}\right) \tau^{3}\right] \ln \frac{M_{\pi^{0}}}{\lambda} \\
& +\left[4 f_{1} e^{2} M_{\pi^{+}}^{2}+e^{2} f_{2} M_{\pi^{+}}^{2} \tau^{3}\right] \ln \frac{M_{\pi^{+}}}{M_{\pi^{0}}} \\
& \tilde{\Sigma}_{6}^{(4, e m)}(\omega)=\left\{-\left(-2 \omega^{2}-l^{2}-8 c_{1} m M_{\pi^{0}}^{2}-m_{\gamma}^{2}\right)\left(\ln \frac{m_{\gamma}}{\lambda}+1\right)\right.
\end{aligned}
$$




$$
\begin{aligned}
& \left.+\omega\left(\frac{1}{\sqrt{m_{\gamma}^{2}-\omega^{2}}}\left(\omega^{2}-l^{2}-8 c_{1} m M_{\pi^{0}}^{2}\right)-\sqrt{m_{\gamma}^{2}-\omega^{2}}\right) \arccos \frac{-\omega}{m_{\gamma}}\right\} \\
\tilde{\Sigma}_{7}^{(4, e m)}(\omega)= & \left\{\frac{1}{2} e^{2} m_{\gamma}^{2}\left(h_{2, \gamma}^{r}(\lambda)+h_{3, \gamma}^{r}(\lambda)\right)+\frac{1}{2} e^{2} m_{\gamma}^{2} h_{1, \gamma}^{r}(\lambda) \tau^{3}\right. \\
& +\frac{2}{F_{\pi}^{2}} B\left(m_{u}-m_{d}\right)\left[4 M_{\pi^{0}}^{2} d_{22}^{r}(\lambda)+\omega^{2} d_{97}^{r}(\lambda)\right] \tau^{3} \\
& +B\left(m_{u}-m_{d}\right) e^{2}\left[2 h_{31}^{r}(\lambda)+\left(h_{29}^{r}(\lambda)+h_{30}^{r}(\lambda)\right) \tau^{3}\right] \\
& +\frac{1}{4} e^{4}\left[h_{1}^{r}(\lambda)+h_{2}^{r}(\lambda)+h_{3}^{r}(\lambda)+\left(h_{4}^{r}(\lambda)+h_{5}^{r}(\lambda)\right) \tau^{3}\right] \\
& +\frac{1}{2} e^{2}\left[l^{2}\left[h_{91}^{r}(\lambda)+h_{92}^{r}(\lambda)+h_{95}^{r}(\lambda) \tau^{3}\right]+\omega^{2}\left[h_{93}^{r}(\lambda)+h_{94}^{r}(\lambda)+h_{96}^{r}(\lambda) \tau^{3}\right]\right] \\
& \left.+2 e^{2} M_{\pi^{0}}^{2}\left[h_{34}^{r}(\lambda) \tau^{3}+\left(h_{32}^{r}(\lambda)+h_{33}^{r}(\lambda)\right)\right]\right\},
\end{aligned}
$$

with

$$
\Gamma_{1}=\frac{g_{A}^{2}}{m \Lambda_{\chi}^{2}}, \Gamma_{2}=\frac{1}{\Lambda_{\chi}^{2}}, \Gamma_{3}=\frac{g_{A}^{2}}{\Lambda_{\chi}^{2}}, \Gamma_{4}=\Gamma_{5}=\Gamma_{2}, \Gamma_{6}=-\frac{e^{2}}{m \Lambda_{\chi}^{2}}\left(1+\tau^{3}\right), \Gamma_{7}=-1,
$$

and

$$
\Lambda_{\chi}^{2}=16 \pi^{2} F_{\pi}^{2}
$$

The first two contributions $\Sigma_{1,2}(\omega)$ are due to pion loops with an insertion $\sim C$, i.e. the charged to neutral pion mass difference. The next two, $\Sigma_{3,4,5}(\omega)$, are pion loops with isospin-violating insertions $\sim c_{5}, f_{1,2,3}$. The photon loop graphs are collected in $\Sigma_{6}(\omega)$ and the last term $\Sigma_{7}(\omega)$ is the contribution from the various strong, virtual photon and "photonic" $\left(\sim m_{\gamma}^{2}\right)$ counterterms at the scale $\lambda$.

\section{Additional fourth order terms}

Here, we enumerate the additional fourth order terms already mentioned in sec. 2.3. We give the number of the monomial $(i=51, \ldots, 90)$, its relativistic and its heavy baryon form,

$$
\begin{aligned}
& \left\langle\left[i \nabla_{\mu}, \tilde{Q}_{+}\right]\left[\tilde{Q}_{-}, u^{\mu}\right]\right\rangle, \quad\left\langle\left[i \nabla_{\mu}, \tilde{Q}_{+}\right]\left[\tilde{Q}_{-}, u^{\mu}\right]\right\rangle, \\
& \left\langle\left[i \nabla_{\mu}, \tilde{Q}_{-}\right]\left[\tilde{Q}_{+}, u^{\mu}\right]\right\rangle, \quad\left\langle\left[i \nabla_{\mu}, \tilde{Q}_{-}\right]\left[\tilde{Q}_{+}, u^{\mu}\right]\right\rangle, \\
& \left\langle\left[i \nabla_{\mu}, \tilde{Q}_{+}\right]\left[\tilde{Q}_{-}, u_{\nu}\right]\right\rangle\left\{\nabla^{\mu}, \nabla^{\nu}\right\}+h . c ., \quad\left\langle\left[i v \cdot \nabla, \tilde{Q}_{+}\right]\left[\tilde{Q}_{-}, v \cdot u\right]\right\rangle, \\
& \left\langle\left[i \nabla_{\mu}, \tilde{Q}_{-}\right]\left[\tilde{Q}_{+}, u_{\nu}\right]\right\rangle\left\{\nabla^{\mu}, \nabla^{\nu}\right\}+h . c ., \quad\left\langle\left[i v \cdot \nabla, \tilde{Q}_{-}\right]\left[\tilde{Q}_{+}, v \cdot u\right]\right\rangle, \\
& \left\langle\left[i \nabla_{\mu}, u_{\nu}\right]\left[\tilde{Q}_{+}, \tilde{Q}_{-}\right]\right\rangle\left\{\nabla^{\mu}, \nabla^{\nu}\right\}+\text { h.c. }, \quad\left\langle[i v \cdot \nabla, v \cdot u]\left[\tilde{Q}_{+}, \tilde{Q}_{-}\right]\right\rangle, \\
& \left\langle\tilde{Q}_{-}\left[\nabla^{\mu},\left[\nabla_{\mu}, \tilde{Q}_{-}\right]\right]\right\rangle, \quad\left\langle\tilde{Q}_{-}\left[\nabla^{\mu},\left[\nabla_{\mu}, \tilde{Q}_{-}\right]\right]\right\rangle, \\
& \left\langle\tilde{Q}_{-}\left[\nabla_{\mu},\left[\nabla_{\nu}, \tilde{Q}_{-}\right]\right]\right\rangle\left\{\nabla^{\mu}, \nabla^{\nu}\right\}+h . c ., \quad\left\langle\tilde{Q}_{-}\left[v \cdot \nabla,\left[v \cdot \nabla, \tilde{Q}_{-}\right]\right]\right\rangle, \\
& \left.\left.\left\langle\left[\nabla_{\mu}, \tilde{Q}_{-}\right]\left[\nabla^{\mu}, \tilde{Q}_{-}\right]\right]\right\rangle, \quad\left\langle\left[\nabla_{\mu}, \tilde{Q}_{-}\right]\left[\nabla^{\mu}, \tilde{Q}_{-}\right]\right]\right\rangle,
\end{aligned}
$$$$
57
$$$$
58
$$ 
$\left.\left\langle\left[\nabla_{\mu}, \tilde{Q}_{-}\right]\left[\nabla_{\nu}, \tilde{Q}_{-}\right]\right]\right\rangle\left\{\nabla^{\mu}, \nabla^{\nu}\right\}+$ h.c.,$\left.\quad\left\langle\left[v \cdot \nabla, \tilde{Q}_{-}\right]\left[v \cdot \nabla, \tilde{Q}_{-}\right]\right]\right\rangle$, $\left.\left\langle Q_{+}\right\rangle\left[i \nabla_{\mu}, \tilde{Q}_{-}\right], u^{\mu}\right], \quad\left\langle Q_{+}\right\rangle\left[\left[i \nabla_{\mu}, \tilde{Q}_{-}\right], u^{\mu}\right]$,

$$
\begin{array}{ll}
\left.\left\langle Q_{+}\right\rangle\left[i \nabla_{\mu}, \tilde{Q}_{-}\right], u_{\nu}\right]\left\{\nabla^{\mu}, \nabla^{\nu}\right\}+\text { h.c. }, & \left\langle Q_{+}\right\rangle\left[\left[i v \cdot \nabla, \tilde{Q}_{-}\right], v \cdot u\right], \\
\left\langle Q_{+}\right\rangle\left[\left[i \nabla_{\mu}, u_{\nu}\right], \tilde{Q}_{-}\right]\left\{\nabla^{\mu}, \nabla^{\nu}\right\}+\text { h.c. }, & \left\langle Q_{+}\right\rangle\left[[i v \cdot \nabla, v \cdot u], \tilde{Q}_{-}\right],
\end{array}
$$$$
\left\langle\left[\nabla^{\mu}, \tilde{Q}_{+}\right]\left[\nabla_{\mu}, \tilde{Q}_{+}\right]\right\rangle, \quad\left\langle\left[\nabla^{\mu}, \tilde{Q}_{+}\right]\left[\nabla_{\mu}, \tilde{Q}_{+}\right]\right\rangle,
$$

$$
\begin{aligned}
& \left\langle\left[\nabla_{\mu}, \tilde{Q}_{+}\right]\left[\nabla_{\nu}, \tilde{Q}_{+}\right]\right\rangle\left\{\nabla^{\mu}, \nabla^{\nu}\right\}+h . c^{\prime}, \quad\left\langle\left[v \cdot \nabla, \tilde{Q}_{+}\right]\left[v \cdot \nabla, \tilde{Q}_{+}\right]\right\rangle, \\
& \left\langle\tilde{Q}_{+}\left[\nabla^{\mu},\left[\nabla_{\mu}, \tilde{Q}_{+}\right]\right]\right\rangle, \quad\left\langle\tilde{Q}_{+}\left[\nabla^{\mu},\left[\nabla_{\mu}, \tilde{Q}_{+}\right]\right]\right\rangle,
\end{aligned}
$$

$$
\left\langle\tilde{Q}_{+}\left[\nabla_{\mu},\left[\nabla_{\nu}, \tilde{Q}_{+}\right]\right]\right\rangle\left\{\nabla^{\mu}, \nabla^{\nu}\right\}+\text { h.c. }, \quad\left\langle\tilde{Q}_{+}\left[v \cdot \nabla,\left[v \cdot \nabla, \tilde{Q}_{+}\right]\right]\right\rangle,
$$$$
\left[\nabla^{\mu},\left[\nabla_{\mu}, \tilde{Q}_{+}\right]\right]\left\langle Q_{+}\right\rangle, \quad\left[\nabla^{\mu},\left[\nabla_{\mu}, \tilde{Q}_{+}\right]\right]\left\langle Q_{+}\right\rangle,
$$

$$
\begin{aligned}
& {\left[\nabla_{\mu},\left[\nabla_{\nu}, \tilde{Q}_{+}\right]\left\langle Q_{+}\right\rangle\left\{\nabla^{\mu}, \nabla^{\nu}\right\}+h . c ., \quad\left[v \cdot \nabla,\left[v \cdot \nabla, \tilde{Q}_{+}\right]\right]\left\langle Q_{+}\right\rangle,\right.} \\
& \sigma^{\mu \nu}\left[\nabla_{\mu}, \tilde{Q}_{+}\right]\left\langle\tilde{Q}_{-} u_{\nu}\right\rangle, \quad i v_{\rho} \varepsilon^{\rho \mu \nu \sigma} S_{\sigma}\left[\nabla_{\mu}, \tilde{Q}_{+}\right]\left\langle\tilde{Q}_{-} u_{\nu}\right\rangle,
\end{aligned}
$$

$$
\sigma^{\mu \nu} \tilde{Q}_{+}\left\langle\left[\nabla_{\mu}, \tilde{Q}_{-}\right] u_{\nu}\right\rangle, \quad i v_{\rho} \varepsilon^{\rho \mu \nu \sigma} S_{\sigma} \tilde{Q}_{+}\left\langle\left[\nabla_{\mu}, \tilde{Q}_{-}\right] u_{\nu}\right\rangle,
$$$$
\sigma^{\mu \nu}\left\langle Q_{+}\right\rangle\left\langle\left[\nabla_{\mu}, \tilde{Q}_{-}\right] u_{\nu}\right\rangle, \quad i v_{\rho} \varepsilon^{\rho \mu \nu \sigma} S_{\sigma}\left\langle Q_{+}\right\rangle\left\langle\left[\nabla_{\mu}, \tilde{Q}_{-}\right] u_{\nu}\right\rangle,
$$$$
\sigma^{\mu \nu}\left[\nabla_{\nu}, \tilde{Q}_{-}\right]\left\langle\tilde{Q}_{+} u_{\nu}\right\rangle, \quad i v_{\rho} \varepsilon^{\rho \mu \nu \sigma} S_{\sigma}\left[\nabla_{\nu}, \tilde{Q}_{-}\right]\left\langle\tilde{Q}_{+} u_{\nu}\right\rangle,
$$$$
\sigma^{\mu \nu} \tilde{Q}_{-}\left\langle\left[\nabla_{\mu}, \tilde{Q}_{+}\right] u_{\nu}\right\rangle, \quad i v_{\rho} \varepsilon^{\rho \mu \nu \sigma} S_{\sigma} \tilde{Q}_{-}\left\langle\left[\nabla_{\mu}, \tilde{Q}_{+}\right] u_{\nu}\right\rangle,
$$

$$
\begin{array}{ll}
\sigma^{\mu \nu} u_{\mu}\left\langle\left[\nabla_{\nu}, \tilde{Q}_{+}\right] \tilde{Q}_{-}\right\rangle, & i v_{\rho} \varepsilon^{\rho \mu \nu \sigma} S_{\sigma} u_{\mu}\left\langle\left[\nabla_{\nu}, \tilde{Q}_{+}\right] \tilde{Q}_{-}\right\rangle, \\
\sigma^{\mu \nu} u_{\mu}\left\langle\left[\nabla_{\nu}, \tilde{Q}_{-}\right] \tilde{Q}_{+}\right\rangle, & i v_{\rho} \varepsilon^{\rho \mu \nu \sigma} S_{\sigma} u_{\mu}\left\langle\left[\nabla_{\nu}, \tilde{Q}_{-}\right] \tilde{Q}_{+}\right\rangle,
\end{array}
$$$$
i \sigma^{\mu \nu}\left[\left[\nabla_{\mu}, \tilde{Q}_{+}\right],\left[\nabla_{\nu}, \tilde{Q}_{+}\right]\right], \quad v_{\rho} \varepsilon^{\rho \mu \nu \sigma} S_{\sigma}\left[\left[\nabla_{\mu}, \tilde{Q}_{+}\right],\left[\nabla_{\nu}, \tilde{Q}_{+}\right]\right],
$$

$$
\begin{array}{ll}
i \sigma^{\mu \nu}\left[\left[\nabla_{\mu}, \tilde{Q}_{-}\right],\left[\nabla_{\nu}, \tilde{Q}_{-}\right]\right], & v_{\rho} \varepsilon^{\rho \mu \nu \sigma} S_{\sigma}\left[\left[\nabla_{\mu}, \tilde{Q}_{-}\right],\left[\nabla_{\nu}, \tilde{Q}_{-}\right]\right], \\
i \sigma^{\mu \nu}\left[\tilde{Q}_{+},\left[\nabla_{\mu},\left[\nabla_{\nu}, \tilde{Q}_{+}\right]\right]\right], & v_{\rho} \varepsilon^{\rho \mu \nu \sigma} S_{\sigma}\left[\tilde{Q}_{+},\left[\nabla_{\mu},\left[\nabla_{\nu}, \tilde{Q}_{+}\right]\right]\right],
\end{array}
$$

$i \sigma^{\mu \nu}\left[\tilde{Q}_{-},\left[\nabla_{\mu},\left[\nabla_{\nu}, \tilde{Q}_{-}\right]\right]\right], \quad v_{\rho} \varepsilon^{\rho \mu \nu \sigma} S_{\sigma}\left[\tilde{Q}_{-},\left[\nabla_{\mu},\left[\nabla_{\nu}, \tilde{Q}_{-}\right]\right]\right]$,

$\gamma^{\mu} \gamma_{5}\left\langle Q_{+}\right\rangle\left[\left[\nabla_{\mu}, \tilde{Q}_{+}\right], u_{\nu}\right] \nabla^{\nu}+$ h.c.,$\quad\left\langle Q_{+}\right\rangle\left[\left[S \cdot \nabla, \tilde{Q}_{+}\right], v \cdot u\right]$,

$\gamma^{\nu} \gamma_{5}\left\langle Q_{+}\right\rangle\left[\left[\nabla_{\mu}, \tilde{Q}_{+}\right], u_{\nu}\right] \nabla^{\mu}+$ h.c.,$\quad\left\langle Q_{+}\right\rangle\left[\left[v \cdot \nabla, \tilde{Q}_{+}\right], S \cdot u\right]$,

$\gamma^{\mu} \gamma_{5}\left\langle\left[\nabla_{\mu}, \tilde{Q}_{+}\right]\left[\tilde{Q}_{+}, u_{\nu}\right]\right\rangle \nabla^{\nu}+$ h.c.,$\quad\left\langle\left[S \cdot \nabla, \tilde{Q}_{+}\right]\left[\tilde{Q}_{+}, v \cdot u\right]\right\rangle$,

$\gamma^{\nu} \gamma_{5}\left\langle\left[\nabla_{\mu}, \tilde{Q}_{+}\right]\left[\tilde{Q}_{+}, u_{\nu}\right]\right\rangle \nabla^{\mu}+$ h.c.,$\quad\left\langle\left[v \cdot \nabla, \tilde{Q}_{+}\right]\left[\tilde{Q}_{+}, S \cdot u\right]\right\rangle$,

$\gamma^{\mu} \gamma_{5}\left\langle\left[\nabla_{\mu}, \tilde{Q}_{-}\right]\left[\tilde{Q}_{-}, u_{\nu}\right]\right\rangle \nabla^{\nu}+$ h.c.,$\quad\left\langle\left[S \cdot \nabla, \tilde{Q}_{-}\right]\left[\tilde{Q}_{-}, v \cdot u\right]\right\rangle$,

$\gamma^{\nu} \gamma_{5}\left\langle\left[\nabla_{\mu}, \tilde{Q}_{-}\right]\left[\tilde{Q}_{-}, u_{\nu}\right]\right\rangle \nabla^{\mu}+$ h.c.,$\quad\left\langle\left[v \cdot \nabla, \tilde{Q}_{-}\right]\left[\tilde{Q}_{-}, S \cdot u\right]\right\rangle$,

$\gamma^{\nu} \gamma_{5}\left\langle\left[\nabla_{\mu}, \tilde{Q}_{+}\right]\left[\nabla_{\nu}, \tilde{Q}_{-}\right]\right\rangle \nabla^{\mu}+\nabla^{\prime} . c ., \quad\left\langle\left[i v \cdot \nabla, \tilde{Q}_{+}\right]\left[S \cdot \nabla, \tilde{Q}_{-}\right]\right\rangle$,

$87 \gamma^{\mu} \gamma_{5}\left\langle\left[\nabla_{\mu}, \tilde{Q}_{+}\right]\left[\nabla_{\nu}, \tilde{Q}_{-}\right]\right\rangle i \nabla^{\nu}+$ h.c.,$\quad\left\langle\left[i S \cdot \nabla, \tilde{Q}_{+}\right]\left[v \cdot \nabla, \tilde{Q}_{-}\right]\right\rangle$,

$i \sigma^{\mu \nu} \gamma_{5}\left\langle\left[\nabla_{\mu},\left[\nabla_{\nu}, \tilde{Q}_{+}\right]\right] \tilde{Q}_{-}\right\rangle, \quad i v^{\mu} S^{\nu}\left\langle\left[\nabla_{\mu},\left[\nabla_{\nu}, \tilde{Q}_{+}\right]\right] \tilde{Q}_{-}\right\rangle$,

$i \sigma^{\mu \nu} \gamma_{5}\left\langle\left[\nabla_{\mu},\left[\nabla_{\nu}, \tilde{Q}_{-}\right]\right] \tilde{Q}_{+}\right\rangle, \quad i v^{\mu} S^{\nu}\left\langle\left[\nabla_{\mu},\left[\nabla_{\nu}, \tilde{Q}_{-}\right]\right] \tilde{Q}_{+}\right\rangle$,

$i \sigma^{\mu \nu} \gamma_{5}\left\langle Q_{+}\right\rangle\left[\nabla_{\mu},\left[\nabla_{\nu}, \tilde{Q}_{-}\right]\right], \quad i v^{\mu} S^{\nu}\left\langle Q_{+}\right\rangle\left[\nabla_{\mu},\left[\nabla_{\nu}, \tilde{Q}_{-}\right]\right]$.

\section{References}


[1] G. Ecker, J. Gasser, A. Pich and E. de Rafael, Nucl. Phys. B321 (1989) 311.

[2] R. Urech, Nucl. Phys. B433 (1995) 234.

[3] H. Neufeld and H. Rupertsberger, Z. Phys. C68 (195) 91.

[4] R. Bauer, J. Kambor and D. Wyler, Nucl. Phys. B460 (1996) 127.

[5] Ulf-G. Meißner, G. Müller and S. Steininger, Phys. Lett. B406 (1997) 154, (E) ibid B407 (1997) 434.

[6] M. Knecht and R. Urech, Nucl. Phys. B519 (1998) 329.

[7] S. Weinberg, Trans. N.Y. Acad. of Sci. 38 (1977) 185.

[8] Ulf-G. Meißner and S. Steininger, Phys. Lett. B419 (1998) 403.

[9] N. Fettes, Ulf-G. Meißner and S. Steininger, hep-ph/9811366, Phys. Lett. B (in press).

[10] V. Bernard, N. Kaiser and Ulf-G. Meißner, Nucl. Phys. A615 (1997) 483.

[11] J. Gasser and H. Leutwyler, Nucl. Phys. B250 (1985) 465.

[12] E. Jenkins and A.V. Manohar, Phys. Lett. B255 (1991) 558.

[13] V. Bernard, N. Kaiser, J. Kambor and Ulf-G. Meißner, Nucl. Phys. B388 (1992) 315.

[14] J. Gasser, M.E. Sainio and A. Švarc, Nucl. Phys. B307 (1988) 779.

[15] V. Bernard, N. Kaiser and Ulf-G. Meißner, Nucl. Phys. A611 (1996) 429.

[16] T. Becher and H. Leutwyler, hep-ph/9901384.

[17] V. Bernard, N. Kaiser and Ulf-G. Meißner, Nucl. Phys. B383 (1992) 442.

[18] V. Bernard, N. Kaiser and Ulf-G. Meißner, Z. Phys. C 70 (1996) 483.

[19] N. Fettes, Ulf-G. Meißner and S. Steininger, Nucl. Phys. A640 (1998) 199.

[20] G. Ecker and M. Mojžiš, Phys. Lett. B365 (1996) 312.

[21] V. Bernard, N. Kaiser and Ulf-G. Meißner, Int. J. Mod. Phys. E4 (1995) 193.

[22] A. Krause, Helv. Phys. Acta 63 (1990) 3.

[23] G. Ecker, Phys. Lett. B336 (1994) 508.

[24] G. Müller and Ulf-G. Meißner, Nucl. Phys. B492 (1997) 379.

[25] Ulf-G. Meißner, G. Müller and S. Steininger, hep-ph/9809446, Ann. Phys. (NY), in press.

[26] N. Fettes, Ulf-G. Meißner, G. Müller and S. Steininger, in preparation.

[27] M. Mojžiš, in preparation.

[28] N. Fettes, Ulf-G. Meißner and S. Steininger, J. High Energy Phys. 9809 (1998) 008.

[29] R. Baur and R. Urech, Nucl. Phys. B499 (1997) 319.

[30] L.S. Brown, W.J. Pardee and R.D. Peccei, Phys. Rev. D4 (1971) 2801.

[31] J. Gasser. H. Leutwyler and M.E. Sainio, Phys. Lett. B253 (1991) 252.

[32] V. Bernard, N. Kaiser and Ulf-G. Meißner, Phys. Lett. B389 (1996) 144.

[33] A.M. Bernstein, Phys. Lett. B442 (1998) 20. 


\section{Figures}
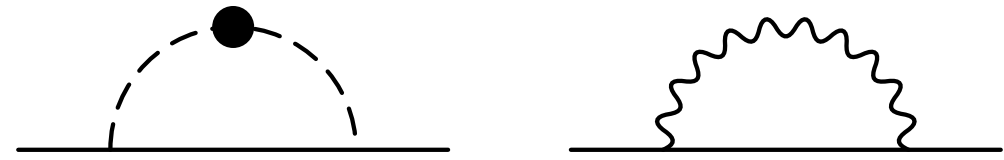

Figure 1: One loop graphs contributing to the nucleons' electromagnetic selfenergy correction. Solid, dashed and wiggly lines denote nucleons, pions and virtual photons, in order. The heavy dot is an insertion $\sim C$ (iterated to all orders).

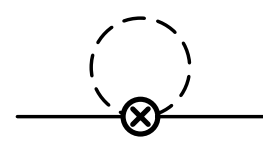

a)

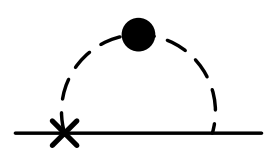

d)

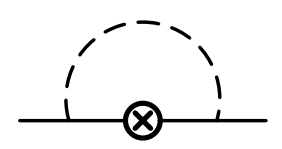

b)

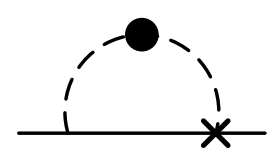

e)

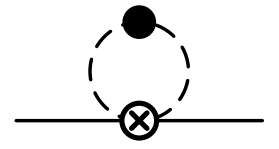

c)

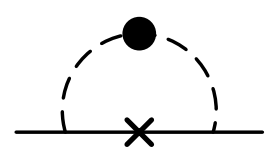

f)

Figure 2: Pion loop contributions to the fourth order em (IV) nucleon self-energy. The circle-cross (cross) denotes an insertion $\sim c_{1}, c_{5}, f_{1}, f_{2}(\sim 1 / m)$. For other notations, see fig.1. 


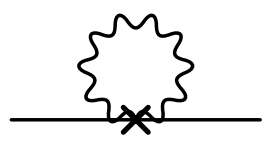

a)

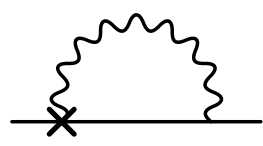

c)

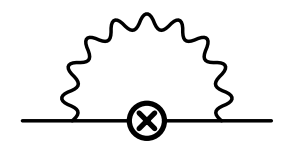

b)

Figure 3: Photon loop contributions to the fourth order em (IV) nucleon selfenergy. For notations, see figs.1,2.
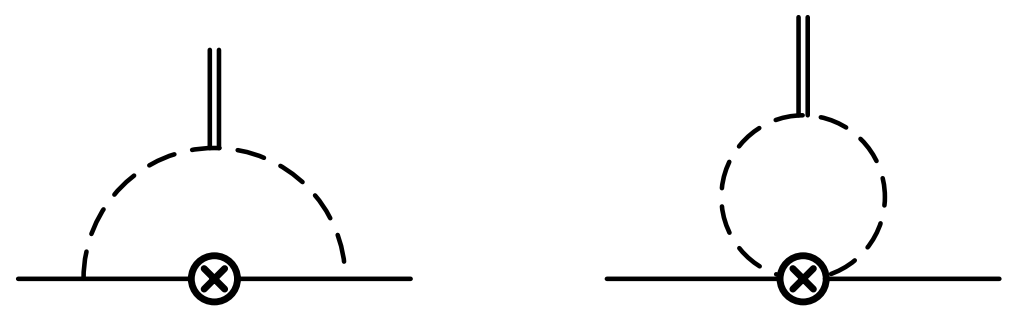

Figure 4: One loop graphs contributing to the electromagnetic (IV) self-energy correction for the scalar form factor of the nucleon. The circle-cross denotes an insertion $\sim c_{5}, f_{2}$. For other notations, see fig.1. 

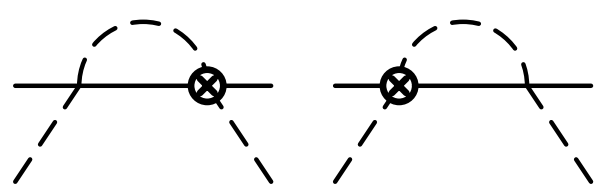

1)

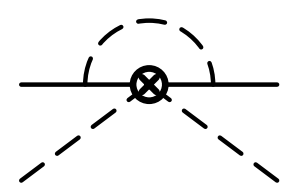

4)
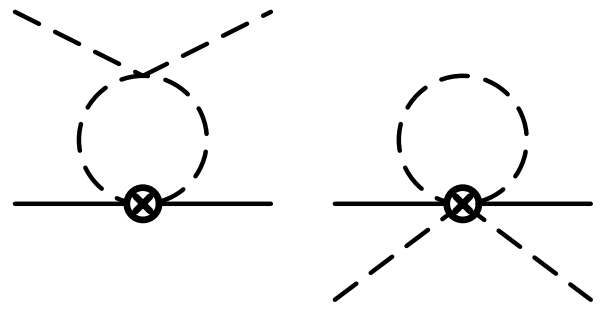

2)

3)

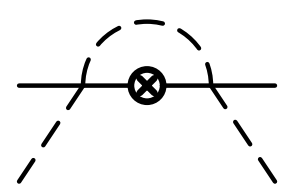

5)

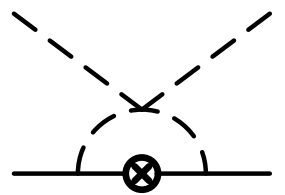

6)

Figure 5: One loop graphs contributing to the fourth order correction to the scattering lengths difference $a\left(\pi^{0} p\right)-a\left(\pi^{0} n\right)$. For notations see fig.1. 